\title{
SOME DEVELOPMENTS IN THE THEORY OF NUMERATIONS $\left({ }^{1}\right)$
}

\author{
BY
}

A. H. KRUSE

1. Introduction. After A. Lindebaum and A. Tarski [4], for each power $\mathrm{m}$ (cf. definition of power later in this section) let $H(\mathrm{~m})$ be the continuum hypothesis statement that there is no power $\mathfrak{n}$ such that $\mathfrak{m}<\mathfrak{n}<2^{\mathfrak{m}}$. Lindenbaum and Tarski stated without proof that if $H(\mathfrak{m}), H\left(2^{\mathfrak{m}}\right)$, and $H\left(2^{2^{\mathfrak{m}}}\right)$, then $2^{2^{m}}$ is the power of a well-ordered set; they stated also without proof that if $H\left(\mathfrak{m}^{2}\right)$ and $H\left(2^{\mathfrak{m}}\right)$, then $2^{\mathfrak{m}^{2}}$ is the power of a well-ordered set. W. Sierpiński [5] proved that if $H(\mathfrak{m}), H\left(2^{\mathfrak{m}}\right)$, and $H\left(2^{2^{\mathfrak{m}}}\right)$, then $\mathfrak{m}$ is the power of a wellordered set, and E. Specker [6] sharpened both results of Lindenbaum and Tarski by proving that if $H(\mathfrak{m})$ and $H\left(2^{\mathfrak{m}}\right)$, then $2^{\mathfrak{m}}$ is the power of a wellordered set.

This paper has arisen from attempts to sharpen Specker's result, which is sharpened in 7.1 and 7.2 (first cf. the definition of $H(\mathfrak{m} ; \alpha)$ in $\$ 7$ prior to 7.1). The writer's efforts along these lines led to developments in the theory of numerations (defined in the first paragraph of \$2) of independent interest, and most of this paper is concerned with these developments.

The content of this paper may be developed in an axiomatic set theory of the von Neumann-Bernays-Gödel kind (cf., e.g., [1]) modified as follows to allow (but not to imply the existence of) elements which are not sets. Each object is either an element or a class. A set is any element which is a class. An atom is an element which is not a class. The usual axioms may be modified in the obvious way to accommodate atoms. We shall assume all the usual axioms so modified except the restrictive axiom and the axiom of choice (cf. \$7).

We indicate briefly our use of some terminology and notation. Elements $x, y$ determine the ordered pair $[x, y]=\{\{x\},\{x, y\}\}$. If $Q$ and $B$ are classes, $a \times B=\{[x, y] \mid x \in a, y \in B\}$. Relations and functions are classes of ordered pairs satisfying (in the case of functions) the usual conditions. If $R$ is a relation, $\operatorname{dom}(R)$ and $\operatorname{im}(R)$ are respectively the domain and the image of $R$ defined in the usual way, and $R^{-1}$ is the inverse (or converse) of $R$. A function from $a$ to (or into) $B$ has domain $Q$ and image $C B$; a function onto $B$ has image $B$. If $\phi$ and $\psi$ are functions, $\psi \circ \phi=\{[x, y] \mid y=\psi(z)$ and $z=\phi(x)$ for some $z\}$. If $\phi, \psi, \xi$ are functions, $\xi \circ \psi \circ \phi=(\xi \circ \psi) \circ \phi=\xi \circ(\psi \circ \phi)$. Each ordinal number will consist of all its predecessors, and a cardinal number is

Received by the editors March 21, 1960.

(1) The research in this paper was partially sponsored by National Science Foundation Research Grant NSF-G4917. 
any ordinal number not equi-potent with any of its members. The first ordinal number is 0 , the void set; $\omega=\boldsymbol{N}_{0}$ is the first infinite ordinal number. A power is a maximal class of mutually equi-potent sets. If $X$ is a set, $|X|$ is the power of $X$ and is the unique power $m$ for which $X \in \mathfrak{m}$. If $a$ and $B$ are classes, then $P(a)$ is the class of all subsets of $a$, and $a \backslash B$ is the relative complement of $B$ in $Q$. The binary connectives $<, \varangle$, etc. will be applied to ordinal numbers and to powers in the usual way. After Tarski [4], $|X|$ $\varliminf^{*}|Y|$ will mean that there is a function from $Y$ onto $X$; if $|X| \leqq *|Y|$, then $2^{|x|} \leqq 2^{|Y|}$. Other terminology and notations will be introduced as they arise or will not be introduced at all if the reader may safely rely on convention and context.

Many proofs and definitions in this paper are by transfinite induction. In such a proof (or definition) the induction step usually will be carried through without being explicitly announced (the induction will be announced), and the induction hypothesis, the statement of which in each case will be obvious, will be referred to and used without being formally introduced. In some cases we shall define for each ordinal number $\mu$ a function $\phi^{\mu}$ whose domain is not a set (but a class). In such a case the functions $\phi^{\mu}$, if defined by induction, must be defined in pieces which are sets, the pieces to be assembled later into the function $\phi^{\mu}$. The ultimate reason for this is the fact that a class which is not a set is not a member of a class; thus a sequence (existing in the theory as a function) of classes which are not sets is out of the question. For an example, cf. the definition of the functions $\Lambda^{\mu}$ in $\$ 3$.

2. The functions $w^{\mu}$. After Bernays $[1, I V$, pp. 140-141] we define a numeration to be any one-one function whose domain is an ordinal number. Throughout the rest of this paper, $\mathcal{u}$ will be the class of all elements, $\theta$ will be the class of all ordinal numbers, $\Re$ will be the class of all numerations, and $\mathcal{U}^{*}=\dot{\mathcal{U}} \backslash \mathfrak{x}$. For each $f \in \mathfrak{N},\{[f(\alpha), f(\beta)] \mid \alpha<\beta \in \operatorname{dom}(f)\}$ is a reflexive wellordering of $\operatorname{im}(f)$.

For each set $X$ we define $w^{\mu}(X)$ for each ordinal number $\mu$ by transfinite induction as follows (2.1-2.4).

2.1. $W^{\circ}(X)=X$.

2.2. $W^{1}(X)=W(X)=\{f \in \Re \mid \operatorname{im}(f) \subset X\}$. It is easy to show that $w(X)$ is a set (cf. 6.12).

2.3. $\left.w^{\mu+1}(X)=w_{\left(W^{\mu}\right.}(X)\right)$. [This holds for $\mu=0$ by 2.1 and 2.2.]

2.4. If $\mu$ is a limit ordinal,

$$
W^{\mu}(X)=\underset{\lambda<\mu}{\bigcup} W^{\lambda}(X)
$$

2.5. REMARK. For each set $X$ and all ordinal numbers $\mu$ and $\nu$,

$$
W^{\nu}\left(W^{\mu}(X)\right) \subset W^{\mu+\nu}(X),
$$

and, if $\nu$ is finite or $\mu=0$ or $\mu$ is a limit ordinal, then

$$
W^{\nu}\left(W^{\mu}(X)\right)=W^{\mu+\nu}(X) .
$$


For each ordinal $\mu, w^{\mu}=\left\{\left[X, w^{\mu}(X)\right] \mid X \in \mathcal{P}(\mathcal{U})\right\}$ is a function with domain $\mathcal{P}(\mathcal{U})$. The lemma below follows by transfinite induction. ber $\mu$.

2.6. Lemma. If $A \subset X \in \odot(\mathcal{U})$, then $w^{\mu}(A) \subset w^{\mu}(X)$ for each ordinal num-

\subsection{Corollary. If $X \in \mathcal{}(\mathcal{u})$,}

$$
W^{\mu}(X)=\bigcup_{A \in \mathcal{P}_{(X)}} W^{\mu}(A)
$$

for each ordinal number $\mu$.

For each class $a$, we may define

$$
W^{\mu}(Q)=\bigcup_{A \in \mathcal{P}(Q)} W^{\mu}(A) .
$$

If $a$ is a set, $W^{\mu}(a)$ is the same as before by 2.7 . It should be noted that we are not extending the domain of the function $w^{\mu}\left(\operatorname{dom}\left(w^{\mu}\right)=\odot(\mathcal{U})\right)$, but we are enlarging the scope of usage of the symbol " $W^{\mu n}$ to express a certain predicate $\left(" B=W^{\mu}(a)\right.$ " expresses a relationship among $\left.B, \mu, Q\right)$. Observe that if $a \subset B$, then $w^{\mu}(a) \subset W^{\mu}(B)$ for each ordinal number $\mu$.

2.8. Lemma. Suppose $X \in \mathcal{P}\left(\mathcal{U}^{*}\right), Y \in \mathcal{P}\left(\mathcal{U}^{*}\right), \mu$ and $\nu$ are ordinal numbers, and $f \in W^{\mu}(X) \cap W^{\prime}(Y)$. Then for each ordinal number $\lambda, f \in W^{\lambda}(X)$ if and only if $f \in w^{\lambda}(Y)$ and also if and only if $f \in w^{\lambda}(X \cap Y)$.

Proof. We proceed by induction on $\min (\mu, \nu)=\mu \bigcap \nu$. We may suppose $f \notin W^{\lambda}(X)$ for all $\lambda<\mu$ and $f \notin W^{\lambda}(Y)$ for all $\lambda<\nu$. Then neither $\mu$ nor $\nu$ is a limit ordinal (cf. 2.4). We may also suppose $\mu \leqq \nu$ (i.e., $\mu \subset \nu$ ).

Suppose first that $\mu=0$. Then $f \in X \subset \mathcal{U}^{*}$ by 2.1 , and $\nu$ has no predecessor (for $f \notin \Re$; cf. 2.2 and 2.3). Hence $\nu=0$, and $f \in Y$. Moreover, $f \in W^{\lambda}(X)$ if and only if $\lambda=0$ or $\lambda$ is a limit ordinal, and similar statements hold for $Y$ and $X \cap Y$ replacing $X$.

Suppose next that $\mu=1$. Then $\operatorname{im}(f) \subset X=w^{\circ}(X)$ by 2.2 and 2.1 , and either $\nu=0$ or $\operatorname{im}(f) \subset w^{\nu-1}(Y)$ by 2.3 and 2.2. Since $1=\mu \leqq \nu, \operatorname{im}(f) \subset w^{\nu-1}(Y)$. The preceding paragraph with $f$ replaced by a member of $\operatorname{im}(f)$ shows that $\nu-1=0$, i.e., $\nu=1$. Then $\operatorname{im}(f) \subset Y$, and $\operatorname{im}(f) \subset X \cap Y$. Moreover, $f \in w^{\lambda}(X)$ if and only if $\lambda=1$ or $\lambda$ is a limit ordinal or both $\lambda$ is not a limit ordinal and $\lambda-1$ is a limit ordinal or both $\lambda>0$ and $\operatorname{dom}(f)=0$; similar statements hold for $Y$ and $X \cap Y$ replacing $X$.

Suppose $\mu>1$. Then $\operatorname{im}(f) \subset w^{\mu-1}(X) \cap w^{\nu-1}(Y)$ by 2.3 and 2.2. By the induction hypothesis, for each $\alpha \in \operatorname{dom}(f)$ and each ordinal number $\lambda, f(\alpha)$ $\in w^{\lambda}(X)$ if and only if $f(\alpha) \in w^{\lambda}(Y)$ and also if and only if $f(\alpha) \in w^{\lambda}(X \cap Y)$. From this it follows easily (by transfinite induction) that for each ordinal number $\lambda, f \in W^{\lambda}(X)$ if and only if $f \in W^{\lambda}(Y)$ and also if and only if $f \in w^{\lambda}(X \cap Y)$. 
The induction on $\min (\mu, \nu)$ is complete. Q.e.d.

2.9. Corollary. Suppose $a \subset \mathcal{U}^{*}$ and $B \subset \mathcal{U}^{*}$. Then for each ordinal number $\mu, w^{\mu}(Q \cap B)=w^{\mu}(Q) \cap w^{\mu}(B)$.

The following lemma may be proved by induction.

2.10. Lemma. Suppose $X$ is a set and $\mu$ a limit ordinal number. Then for each element $f, f \in W^{\mu}(X)$ if and only if $f \in W^{\lambda}(X)$ for some nonlimit ordinal $\lambda<\mu$.

3. The functions $\Lambda^{\mu}$. For each set $X$ we define the function $\Lambda_{X}^{\mu}$ with domain $W^{\mu}(X)$ and image $C W(X)$ for each ordinal $\mu$ by transfinite induction as follows (3.1-3.6).

3.1. $\Lambda_{X}^{0}(x)=\{[0, x]\}$ (i.e., $\operatorname{dom}\left(\Lambda_{X}^{0}(x)\right)=\{0\}=1$ and $\left.\Lambda_{X}^{0}(x)(0)=x\right)$ for each $x \in X=w^{\circ}(X)$.

3.2. $\Lambda_{X}^{1}(f)=f$ for each $f \in w^{1}(X)$.

3.3. Suppose $\mu>1$ is not a limit ordinal. Suppose $f \in w^{\mu}(X)$. Define the relation $S_{f} \subset X \times X$ as follows. For all $x, y \in X$, let $x S_{f} y$ if and only if there are $g, h$ $\in \operatorname{im}(f)$ such that $f^{-1}(g)$ is the smallest $\alpha \in \operatorname{dom}(f)$ for which $x \in \operatorname{im}\left(\Lambda_{X}^{\mu-1}(f(\alpha))\right)$, such that $f^{-1}(h)$ is the smallest $\alpha \in \operatorname{dom}(f)$ for which $y \in \operatorname{im}\left(\Lambda_{X}^{\mu-1}(f(\alpha))\right)$, such that $f^{-1}(g) \leqq f^{-1}(h)$, and such that

$$
\left(\Lambda_{X}^{\mu-1}(g)\right)^{-1}(x) \leqq\left(\Lambda_{X}^{\mu-1}(g)\right)^{-1}(y)
$$

if $g=h$. There is a unique function $\Lambda_{X}^{\mu}(f) \in W(X)$ such that $\operatorname{im}\left(\Lambda_{X}^{\mu}(f)\right)$ $=\operatorname{dom}\left(S_{f}\right)$ and such that $\alpha \leqq \beta \in \operatorname{dom}\left(\Lambda_{X}^{\mu}(f)\right)$ implies

$$
\left[\Lambda_{X}^{\mu}(f)(\alpha), \Lambda_{X}^{\mu}(f)(\beta)\right] \in S_{f} .
$$

[Observe from 3.1 and 3.2 that if " $\mu>1$ " is replaced by " $\mu=1$," the procedure in 3.3 gives the same $\Lambda_{X}^{\mu}(f)$ defined in 3.2.]

3.4. If $\mu$ is a limit ordinal and $f \in W^{\mu}(X)$, then, where $\alpha$ is the first $\lambda<\mu$ for which $f \in w^{\lambda}(X)$ (cf. 2.4), $\Lambda_{X}^{\mu}(f)=\Lambda_{X}^{\alpha}(f)$.

3.5. Theorem. Suppose $X$ is a set and $\mu>0$ is an ordinal number. Then $\operatorname{im}\left(\Lambda_{X}^{\mu}\right)=W(X)$.

Proof. We proceed by induction. For $\mu=1$, apply 3.2.

Consider $\mu>1$ not a limit ordinal. Consider $f \in W(X)$. By the induction hypothesis there is $g \in W^{\mu-1}(X)$ such that $\Lambda_{X}^{\mu-1}(g)=f$. Then $\{[0, g]\} \in W^{\mu}(X)$, and, by 3.3, $\Lambda_{X}^{\mu^{\prime}}(\{[0, g]\})=\Lambda_{X}^{\mu-1}(g)=f$. Thus $\operatorname{im}\left(\Lambda_{X}^{\mu}\right)=W(X)$.

The induction step for $\mu$ a limit ordinal is trivial (cf. 3.4). Q.e.d.

3.6. Lemma. Suppose $X$ and $Y$ are sets, $\mu$ is an ordinal number, and $f \in W^{\mu}(X) \cap w^{\mu}(Y)$. If $\mu$ is finite or $X \cup Y \subset \mathcal{U}^{*}$, then $\Lambda_{X}^{\mu}(f)=\Lambda_{Y}^{\mu}(f)$.

Proof. We proceed by induction on $\mu$. For $\mu=0$, apply 3.1. For $\mu=1$, apply 3.2 . 
Consider $\mu>1$ not a limit ordinal. It suffices to show that $S_{f}$ in 3.3 is independent of $X \subset \mathcal{u}^{*}$ for which $f \in w^{\mu}(X)$; this follows easily from the induction hypothesis (with $f$ replaced by $f(\alpha)$ for each $\alpha \in \operatorname{dom}(f)$ ).

Consider $\mu$ a limit ordinal. In this case $\Lambda_{X}^{\mu}(f)=\Lambda_{Y}^{\mu}(f)$ follows from 2.8, 3.4, and the induction hypothesis.

The induction is complete. Q.e.d.

Suppose $\mu$ is an ordinal number. Let $\mathfrak{u}_{1}=\mathcal{u}$ if $\mu$ is finite, and let $\mathfrak{u}_{1}=\mathcal{u}^{*}$ if $\mu$ is infinite. Then for each set $X \subset \mathfrak{u}_{1}$,

$$
\Lambda_{X}^{\mu}=\left\{\left[f, \Lambda_{X}^{\mu}(f)\right] \mid f \in W^{\mu}(X)\right\}
$$

is a function, and, by 3.6 ,

$$
\Lambda^{\mu}=\bigcup_{x \in \mathcal{P}\left(\mathfrak{U}_{1}\right)} \Lambda_{x}^{\mu}
$$

is a function with domain $w^{\mu}\left(\mathfrak{u}_{1}\right)$. For each set $X \subset \mathfrak{u}_{1}$ and each $f \in \mathfrak{w}^{\mu}(X)$, $\Lambda^{\mu}(f)=\Lambda_{X}^{\mu}(f)$.

The following lemma is easily proved from 3.1-3.3. The details will be omitted.

3.7. Lемма. Suppose $X$ is a set, $\mu$ is an ordinal number, and $f \in w^{\mu+1}(X)$. Then

$$
\operatorname{im}\left(\Lambda^{\mu+1}(f)\right)=\underset{\alpha \in \operatorname{dom}(f)}{\bigcup} \operatorname{im}\left(\Lambda^{\mu}(f(\alpha))\right) .
$$

In particular, if $\mu=1$,

$$
\operatorname{im}\left(\Lambda^{2}(f)\right)=\underset{\alpha \in \operatorname{dom}(f)}{U} \operatorname{im}(f(\alpha)) .
$$

3.8. Theorem. Suppose $\mu$ is an ordinal number and $f \in w^{\mu}\left(\mathcal{U}^{*}\right)$. Then for each set $X \subset \mathcal{U}^{*},(\mathrm{i}),(\mathrm{ii})$, and (iii) below are mutually equivalent.

(i) $f \in w^{\mu}(X)$.

(ii) $\Lambda^{\mu}(f) \in W(X)$.

(iii) $\operatorname{im}\left(\Lambda^{\mu}(f)\right) \subset X$.

Thus $\operatorname{im}\left(\Lambda^{\mu}(f)\right)$ is the smallest set $X \subset \mathcal{u}^{*}$ such that $f \in w^{\mu}(X)$. [If $\mu$ is finite, $\mathcal{u}^{*}$ may be replaced consistently by $\mathcal{u}$.]

Proof. Trivially, (i) implies (ii). By 2.2, (ii) is equivalent to (iii). It remains to prove that (ii) or (iii) implies (i).

We proceed by induction on $\mu$. For $\mu=0$ apply 2.1 and 3.1. For $\mu=1$ apply 2.2 and 3.2.

Consider $\mu>\Lambda$ not a limit ordinal. Consider $X \in \mathcal{P}\left(\mathcal{u}^{*}\right)$ such that $\operatorname{im}\left(\Lambda^{\mu}(f)\right) \subset X$. Then $\operatorname{im}\left(\Lambda^{\mu-1}(f(\alpha))\right) \subset X$ and $f(\alpha) \in w^{\mu-1}(X)$ for each $\alpha \in \operatorname{dom}(f)$ by 3.6 and the induction hypothesis. Hence $\left.f \in w^{(} w^{\mu-1}(X)\right)$ $=w^{\mu}(X)$ by 2.2 and 2.3. Thus (iii) implies (i). 
Consider $\mu$ a limit ordinal. Consider $X \in \mathcal{P}\left(\mathcal{U}^{*}\right)$ such that $\operatorname{im}\left(\Lambda^{\mu}(f)\right) \subset X$. By 2.4 there is a first ordinal $\lambda<\mu$ such that $f \in w^{\lambda}\left(\mathcal{U}^{*}\right)$, say $\lambda=\alpha$. Then (cf. 2.8 and 3.4) $\Lambda^{\mu}(f)=\Lambda^{\alpha}(f)$, and $\operatorname{im}\left(\Lambda^{\alpha}(f)\right) \subset X$. By the induction hypothesis, $f \in W^{\alpha}(X)$. By $2.4, f \in W^{\mu}(X)$. Thus (iii) implies (i).

The induction is complete. Q.e.d.

3.9. THEOREM. Suppose $\mu$ and $\nu$ are ordinal numbers, and suppose $f \in w^{\mu}\left(\mathcal{u}^{*}\right)$ $\cap w^{\nu}\left(\mathcal{U}^{*}\right)$. Then $\Lambda^{\mu}(f)=\Lambda^{\nu}(f)$.

Proof. We proceed by induction on $\max (\mu, \nu)=\mu \cup \nu$. We may suppose $\mu<\nu$. Then $\nu>0$. If $\nu=1$, then $\mu=0$, and $f \in \mathcal{u}^{*} \cap \Re=0$, a contradiction. Hence we may suppose $\nu>1$. By 3.4 we may suppose neither $\mu$ nor $\nu$ is a limit ordinal. If $\mu \geqq 1$, then by 3.3 and the induction hypothesis it is easily seen that $\Lambda^{\mu}(f)=\Lambda^{\nu}(f)$. If $\mu=0$, it is easily seen that $\nu$ must be a limit ordinal contrary to supposition. Q.e.d. Then

3.10. Lemma. Suppose $X$ is a set, $\mu$ is an ordinal number, and $f \in w^{\mu+2}(f)$.

$$
\Lambda^{\mu+2}(f)=\Lambda^{\mu+1}\left(\Lambda^{2}(f)\right)
$$

Proof. By 3.2 and 3.3,

(a) $\{f(\alpha)(\lambda) \mid \alpha \in \operatorname{dom}(f)$ and $\lambda \in \operatorname{dom}(f(\alpha))\}=\left\{\Lambda^{2}(f)(\gamma) \mid \gamma \in \operatorname{dom}\left(\Lambda^{2}(f)\right)\right\}$. By 3.7 and (a),

$$
\begin{aligned}
\operatorname{im}\left(\Lambda^{\mu+2}(f)\right) & =\bigcup_{\alpha \in \operatorname{dom}(\gamma)} \operatorname{im}\left(\Lambda^{\mu+1}(f(\alpha))\right) \\
& =\bigcup_{\alpha \in \operatorname{dom}(\gamma)} \bigcup_{\lambda \in \operatorname{dom}(\gamma(\alpha))} \operatorname{im}\left(\Lambda^{\mu}(f(\alpha)(\lambda))\right) \\
& =\bigcup_{\gamma \in \operatorname{dom}\left(\Lambda^{2}(\gamma)\right)} \operatorname{im}\left(\Lambda^{\mu}\left(\Lambda^{2}(f)(\gamma)\right)\right) \\
& =\operatorname{im}\left(\Lambda^{\mu+1}\left(\Lambda^{2}(f)\right)\right) .
\end{aligned}
$$

Let

$$
\begin{aligned}
A & =\operatorname{im}\left(\Lambda^{\mu+2}(f)\right)=\operatorname{im}\left(\Lambda^{\mu+1}\left(\Lambda^{2}(f)\right)\right), \\
R_{1} & =\left\{\left[\Lambda^{\mu+2}(f)(\alpha), \Lambda^{\mu+2}(f)(\beta)\right] \mid \alpha<\beta \in \operatorname{dom}\left(\Lambda^{\mu+2}(f)\right)\right\}, \\
R_{2} & =\left\{\left[\Lambda^{\mu+1}\left(\Lambda^{2}(f)\right)(\alpha), \Lambda^{\mu+1}\left(\Lambda^{2}(f)\right)(\beta)\right] \mid \alpha<\beta \in \operatorname{dom}\left(\Lambda^{\mu+1}\left(\Lambda^{2}(f)\right)\right)\right\} .
\end{aligned}
$$

Then $R_{1} \cup R_{2} \subset A \times A$, and each of $R_{1}$ and $R_{2}$ reflexively well-orders $\mathrm{A}$.

To prove $\Lambda^{\mu+2}(f)=\Lambda^{\mu+1}\left(\Lambda^{2}(f)\right)$ it will suffice to prove $R_{1}=R_{2}$. Using (b) we make definitions (c) and (d) below.

(c) For each $x \in A$, let $\alpha_{x}$ be the first $\alpha \in \operatorname{dom}(f)$ such that $x \in \operatorname{im}\left(\Lambda^{\mu+1}(f(\alpha))\right)$.

(d) For each $x \in A$, let $\gamma_{x}$ be the first $\gamma \in \operatorname{dom}\left(\Lambda^{2}(f)\right)$ such that $x \in \operatorname{im}\left(\Lambda^{\mu}\left(\Lambda^{2}(f)(\gamma)\right)\right)$.

Using (c) and (d) we make definitions (e) and (f) below. 
(e) For each $x \in A$, let $\beta_{x}$ be the first $\beta \in \operatorname{dom}\left(\Lambda^{\mu+1}\left(f\left(\alpha_{x}\right)\right)\right)$ such that $x=\Lambda^{\mu+1}\left(f\left(\alpha_{x}\right)\right)(\beta)$.

(f) For each $x \in A$, let $\delta_{x}$ be the first $\delta \in \operatorname{dom}\left(\Lambda^{\mu}\left(\Lambda^{2}(f)\left(\gamma_{x}\right)\right)\right)$ such that $x=\Lambda^{\mu}\left(\Lambda^{2}(f)\left(\gamma_{x}\right)\right)(\delta)$.

Using (c) and 3.7 we make definition (g) below.

(g) For each $x \in A$, let $\lambda_{x}$ be the first $\lambda \in \operatorname{dom}\left(f\left(\alpha_{x}\right)\right)$ such that $x \in \operatorname{im}\left(\Lambda^{\mu}\left(f\left(\alpha_{x}\right)(\lambda)\right)\right)$.

By (c), (g), and 3.7,

(h) for each $x \in A, f\left(\alpha_{x}\right)\left(\lambda_{x}\right) \notin \operatorname{im}(f(\alpha))$ for each $\alpha<\alpha_{x}$, and $f\left(\alpha_{x}\right)\left(\lambda_{x}\right)$ $\neq f\left(\alpha_{x}\right)(\lambda)$ for each $\lambda<\lambda_{x}$.

By 3.2, 3.3, (h), (c), (g), and (d) (cf. also (a)),

(i) $f\left(\alpha_{x}\right)\left(\lambda_{x}\right)=\Lambda^{2}(f)\left(\gamma_{x}\right)$ for each $x \in A$,

(j) for all $x, y \in A, \gamma_{x}<\gamma_{y}$ if and only if either $\alpha_{x}<\alpha_{y}$ or both $\alpha_{x}=\alpha_{y}$ and $\lambda_{x}<\lambda_{y}$, and $\gamma_{x}=\gamma_{y}$ if and only if both $\alpha_{x}=\alpha_{y}$ and $\lambda_{x}=\lambda_{y}$.

By (f) and (i),

(k) for each $x \in A, \delta_{x}$ is the first $\delta \in \operatorname{dom}\left(\Lambda^{\mu}\left(f\left(\alpha_{x}\right)\left(\lambda_{x}\right)\right)\right)$ such that $x=\Lambda^{\mu}\left(f\left(\alpha_{x}\right)\left(\lambda_{x}\right)\right)\left(\delta_{x}\right)$.

By 3.3, (e), (g), and (k),

(l) $\Lambda^{\mu+1}\left(f\left(\alpha_{x}\right)\left(\beta_{x}\right)\right)=\Lambda^{\mu}\left(f\left(\alpha_{x}\right)\left(\lambda_{x}\right)\right)\left(\delta_{x}\right)$ for each $x \in A$,

(m) for all $x, y \in A$ with $\alpha_{x}=\alpha_{y}, \beta_{x}<\beta_{y}$ if and only if either $\lambda_{x}<\lambda_{y}$ or both $\lambda_{x}=\lambda_{y}$ and $\delta_{x}<\delta_{y}$, and $\beta_{x}=\beta_{y}$ if and only if both $\lambda_{x}=\lambda_{y}$ and $\delta_{x}=\delta_{y}$.

By (c), (e), and 3.2,

(n) for all $x, y \in A, x R_{1} y$ if and only if either $\alpha_{x}<\alpha_{y}$ or both $\alpha_{x}=\alpha_{y}$ and $\beta_{x} \leqq \beta_{y}$.

By (d), (f), and 3.2,

(o) for all $x, y \in A, x R_{2} y$ if and only if either $\gamma_{x}<\gamma_{y}$ or both $\gamma_{x}=\gamma_{y}$ and $\delta_{x} \leqq \delta_{y}$.

It is easily shown from (j), (m), (n), and (o) that $R_{1}=R_{2}$. Q.e.d.

3.11. Theorem. Suppose $X$ is a set, $\mu$ is an ordinal number, $\nu$ is a finite ordinal number, and $f \in W^{\mu+\nu}(f)$. Then

$$
\Lambda^{\mu+\nu}(f)=\Lambda^{\mu+1}\left(\Lambda^{\nu}(f)\right) \text {. }
$$

Proof. For $\nu=0$, apply 3.1 and 3.4 if $\mu>0$, and apply 3.1 and 3.2 if $\mu=0$ (or cf. the comment after the proof of 3.7). For $\nu=1$ apply 3.2. For $\nu=2$, apply 3.10. We now proceed by induction (using 3.10), the induction step being

$$
\Lambda^{\mu+\nu+1}(f)=\Lambda^{\mu+\nu}\left(\Lambda^{2}(f)\right)=\Lambda^{\mu+1}\left(\Lambda^{\nu}\left(\Lambda^{2}(f)\right)\right)=\Lambda^{\mu+1}\left(\Lambda^{\nu+1}(f)\right) .
$$

4. Maps $W^{\mu}(X) \rightarrow W^{\mu}(Y)$ induced by maps $X \rightarrow Y$. Suppose $\phi$ is a function with $\operatorname{dom}(\phi)$ a set. We define $\phi^{\mu}$, a function with domain $w^{\mu}(\operatorname{dom}(\phi))$, for each ordinal number $\mu$ by transfinite induction as follows (4.1-4.4).

4.1. $\phi^{0}=\phi$.

4.2. Suppose $f \in W^{1}(\operatorname{dom}(\phi))$. Let $A=\{\beta \in \operatorname{dom}(f) \mid \phi(f(\beta)) \neq \phi(f(\alpha))$ for 
each $\alpha<\beta$. There is a unique strictly increasing function $\xi$ with $\operatorname{dom}(\xi)$ an ordinal number and $\operatorname{im}(\xi)=A$. Let $\phi^{1}(f)=\phi \circ f \circ \xi$. Then $\operatorname{dom}\left(\phi^{1}(f)\right)=\operatorname{dom}(\xi)$. Thus we obtain a function $\phi^{1}$ with domain $W^{1}(\operatorname{dom}(\phi))$.

4.3. $\phi^{\mu+1}=\left(\phi^{\mu}\right)^{1}$. [This holds for $\mu=0$ by 4.1 and 4.2.]

4.4. Suppose $\mu$ is a limiting ordinal. If $f \in w^{\mu}(\operatorname{dom}(\phi))$, then, where $\alpha$ is the first ordinal $\lambda<\mu$ such that $f \in w^{\lambda}(\operatorname{dom}(\phi))(c f .2 .4)$, let $\phi^{\mu}(f)=\phi^{\alpha}(f)$.

The following theorem may be proved by a straightforward induction, the details of which will be omitted.

4.5. Theorem. Suppose $X$ and $Y$ are sets, suppose $\phi$ is a function from $X$ to $Y$, and suppose $\mu$ is an ordinal number. Then $\phi^{\mu}$ is a function from $w^{\mu}(X)$ to $w^{\mu}(Y)$.

The proof of the following lemma is straightforward (cf. 4.2) and will be omitted.

4.6. Lemma. Suppose $X$ is a set and $\phi$ a function with domain $X$. Then $\operatorname{im}\left(\phi^{1}(f)\right)=\operatorname{im}(\phi \circ f)$ for each $f \in W^{1}(X)$. If $\phi$ is one-one, then $\phi^{1}(f)=\phi \circ f$ for each $f \in W^{1}(X)$.

4.7. Theorem. Suppose $\mathrm{X} \subset \mathcal{U}^{*}$ and $Y$ are sets, and suppose $\phi$ is a function from $X$ to $Y$. Suppose $\mu$ and $\nu$ are ordinal numbers, and suppose $f \in^{\mu}(X)$ $\cap w^{\nu}(X)$. Then $\phi^{\mu}(f)=\phi^{\nu}(f)$.

Proof. We proceed by induction on $\max (\mu, \nu)=\mu \cup \nu$. We may suppose $\mu<\nu$.

Suppose $\nu$ is not a limit ordinal. There is a smallest ordinal $\lambda \leqq \mu$ such that $f \in W^{\lambda}(X)$ and $\phi^{\lambda}(f)=\phi^{\mu}(f)$. By 4.4, $\lambda$ is not a limit ordinal. Since $w^{0}(X)$ $=X \subset \mathcal{U}^{*}$ and $W^{\nu}(X)=W^{\prime}\left(W^{\nu-1}(X)\right) \subset \mathfrak{N}, W^{0}(X) \cap W^{\nu}(X)$ is void. Hence $\lambda \neq 0$. Now $f(\alpha) \in w^{\lambda-1}(X) \cap w^{-1}(X)$ and $\phi^{\lambda-1}(f(\alpha))=\phi^{-1}(f(\alpha))$ for each $\alpha \in \operatorname{dom}(f)$ by the induction hypothesis. It is seen from this and 4.2 that

$$
\phi^{\mu}(f)=\phi^{\lambda}(f)=\left(\phi^{\lambda-1}\right)^{1}(f)=\left(\phi^{\nu-1}\right)^{1}(f)=\phi^{\nu}(f) .
$$

Suppose $\nu$ is a limit ordinal. By $4.4, \phi^{\nu}(f)=\phi^{\beta}(f)$ where $\beta$ is the first ordinal $\lambda<\nu$ for which $f \in w^{\lambda}(X)$. Then

$$
\phi^{\mu}(f)=\phi^{\lambda}(f)=\phi^{\nu}(f)
$$

by the induction hypothesis.

The induction is complete. Q.e.d.

4.8. Theorem. Suppose $X \subset \mathcal{U}^{*}$ and $Y \subset \mathcal{U}^{*}$ are sets and $\phi$ a function from $X$ to $Y$. Suppose $\mu$ is an ordinal number and $f \in W^{\mu}(X)$. Then for each ordinal number $\lambda, f \in W^{\lambda}(X)$ if and only if $\phi^{\mu}(f) \in W^{\lambda}(Y)$.

Proof. We proceed by induction on $\mu$. By 4.7 we may suppose $f \bigoplus^{{ }^{\lambda}}(X)$ for each ordinal $\lambda<\mu$. Then, by $2.4, \mu$ is not a limit ordinal. 
Consider first the case in which $\mu=0$. It is easily seen from $f \notin \Re$ that $f \in W^{\lambda}(X)$ if and only if $\lambda=0$ or $\lambda$ is a limit ordinal and then also, since $\phi^{0}(f)=\phi(f) \notin \Re$, if and only if $\phi^{0}(f) \in W^{\lambda}(Y)$.

Now consider the case in which $\mu>0$. Then $f(\alpha) \in w^{\mu-1}(X)$ for each $\alpha \in \operatorname{dom}(f)$. Suppose first that $\lambda>0$ is a nonlimit ordinal number. Then (a)-(f) below are mutually equivalent. [To prove (a) equivalent to (b) and (e) equivalent to $(f)$, use $2.1-2.4$, especially 2.3 . To prove (b) equivalent to (c), use the induction hypothesis. To prove (c) equivalent to (d), use 4.3. To prove (d) equivalent to (e), use 4.6.]
(a) $f \in w^{\lambda}(X)$.
(b) $f(\alpha) \in w^{\lambda-1}(X)$ for each $\alpha \in \operatorname{dom}(f)$.
(c) $\phi^{\mu-1}(f(\alpha)) \in w^{\lambda-1}(Y)$ for each $\alpha \in \operatorname{dom}(f)$.
(d) $\left(\phi^{\mu-1}\right)^{1}(f)(\beta) \in w^{\lambda-1}(Y)$ for each $\beta \in \operatorname{dom}\left(\left(\phi^{\mu-1}\right)^{1}(f)\right)$.
(e) $\phi^{\mu}(f)(\beta) \in W^{\lambda-1}(Y)$ for each $\beta \in \operatorname{dom}\left(\phi^{\mu}(f)\right)$.
(f) $\phi^{\mu}(f) \in W^{\lambda}(Y)$.

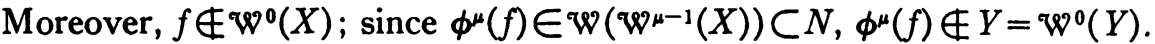
Thus (a) is equivalent to (f) for each nonlimit ordinal number $\lambda$. Then also, by $2.10,(a)$ is equivalent to (f) for each ordinal number $\lambda$.

The induction is complete. Q.e.d.

4.9. Lemma. Suppose $X$ and $Y$ are sets, suppose $\phi$ is a function from $X$ to $Y$, and suppose $\psi$ is a function with domain $Y$. Then $(\psi \circ \phi)^{1}=\psi^{1} \circ \phi^{1}$. Let

Proof. Consider $f \in W^{1}(X)$. Let $A$ and $\xi$ be as in 4.2. Then $\phi^{1}(f)=\phi \circ f \circ \xi$.

$$
B=\left\{\beta \in \operatorname{dom}\left(\phi^{1}(f)\right) \mid \psi\left(\phi^{1}(f)(\beta)\right) \neq \psi\left(\phi^{1}(f)(\alpha)\right) \text { for each } \alpha<\beta\right\} .
$$

There is a unique strictly increasing function $\tau$ with $\operatorname{dom}(\tau)$ an ordinal number and $\operatorname{im}(\tau)=B$. By $4.2, \psi^{1}\left(\phi^{1}(f)\right)=\psi \circ\left(\phi^{1}(f)\right) \circ \tau=\psi \circ \phi \circ f \circ \xi \circ \tau$. Moreover,

$$
B=\{\beta \in \operatorname{dom}(\xi) \mid \psi(\phi(f(\xi(\beta)))) \neq \psi(\phi(f(\xi(\alpha)))) \text { for each } \alpha<\beta\},
$$

and hence (cf. the definitions of $A$ and $\xi$ )

$$
\operatorname{im}(\xi \mid B)=\{\gamma \in \operatorname{dom}(f) \mid(\psi \circ \phi)(f(\gamma)) \neq(\psi \circ \phi)(f(\delta)) \text { for each } \delta<\gamma\} .
$$

Also, $\xi \circ \tau$ is a strictly increasing function with $\operatorname{dom}(\xi \circ \tau)=\operatorname{dom}(\tau)$ and $\operatorname{im}(\xi \circ \tau)=\operatorname{im}(\xi \mid B)$. Hence, by 4.2 ,

$$
(\psi \circ \phi)^{1}(f)=(\psi \circ \phi) \circ f \circ(\xi \circ \tau)=\psi^{1}\left(\phi^{1}(f)\right)=\left(\psi^{1} \circ \phi^{1}\right)(f) .
$$

Thus $(\psi \circ \phi)^{1}=\psi^{1} \circ \phi^{1}$. Q.e.d.

4.10. Theorem. Suppose $X, Y$, and $Z$ are sets, $\phi$ is a function from $X$ to $Y$, and $\psi$ is a function from $Y$ to $Z$. Suppose also that $\mu$ is an ordinal number, and suppose that either $\mu$ is finite or $X \cup Y \cup Z \subset \mathcal{U}^{*}$. Then 4.10.1-4.10.5 below hold. 
4.10.1. $(\psi \circ \phi)^{\mu}=\psi^{\mu} \circ \phi^{\mu}$.

4.10.2. If $\phi(x)=x$ for each $x \in X$, then $\phi^{\mu}(f)=f$ for each $f \in w^{\mu}(X)$.

4.10.3. If $\phi$ maps $X$ one-one and onto $Y$ and $\psi$ is inverse to $\phi$, then $\phi^{\mu}$ maps $W^{\mu}(X)$ one-one and onto $W^{\mu}(Y)$ and $\psi^{\mu}$ is inverse to $\phi^{\mu}$.

4.10.4. If $\phi$ is one-one, then $\phi^{\mu}$ is one-one, $\phi^{\mu+1}(f)=\phi^{\mu} \circ f$ for each $f \in w^{\mu+1}(X)$, and $\operatorname{dom}\left(\phi^{\mu}(f)\right)=\operatorname{dom}(f)$ for each $f \in w^{\mu}(X) \backslash \mathcal{u}^{*}$ if $\mu>0$.

4.10.5. For each $f \in w^{\mu+1}(X), \operatorname{im}\left(\phi^{\mu+1}(f)\right)=\operatorname{im}\left(\phi^{\mu} \circ f\right)$.

4.10.6. For each $f \in w^{\mu}(X), \Lambda^{\mu}\left(\phi^{\mu}(f)\right)=\phi^{1}\left(\Lambda^{\mu}(f)\right)$.

Proof of 4.10.1. We proceed by induction on $\mu$. For $\mu=0$, apply 4.1. For $\mu=1$, apply 4.9 .

Consider the case in which $\mu>1$ is not a limit ordinal. By the induction hypothesis, 4.3 , and 4.9 ,

$$
(\psi \circ \phi)^{\mu}=\left((\psi \circ \phi)^{\mu-1}\right)^{1}=\left(\psi^{\mu-1} \circ \phi^{\mu-1}\right)^{1}=\left(\psi^{\mu-1}\right)^{1} \circ\left(\phi^{\mu-1}\right)^{1}=\psi^{\mu} \circ \phi^{\mu} .
$$

Consider the case in which $\mu$ is a limit ordinal. Consider $f \in w^{\mu}(X)$. There is a first ordinal $\lambda<\mu$ for which $f \in w^{\lambda}(X)$, say $\lambda=\alpha$. By 4.4, the induction hypothesis, and 4.8 or 4.7 ,

$$
(\psi \circ \phi)^{\mu}(f)=(\psi \circ \phi)^{\alpha}(f)=\psi^{\alpha}\left(\phi^{\alpha}(f)\right)=\psi^{\alpha}\left(\phi^{\mu}(f)\right)=\psi^{\mu}\left(\phi^{\mu}(f)\right) .
$$

Thus $(\psi \circ \phi)^{\mu}=\psi^{\mu} \circ \phi^{\mu}$.

The induction is complete. Q.e.d.

The proof of 4.10.2 is a straightforward induction using 4.1-4.4. We omit the details.

By standard arguments, 4.10.3 follows from 4.10.1 and 4.10.2.

Proof of 4.10.4. Suppose $\phi$ is one-one. We may suppose $Y=\operatorname{im}(\phi)$. Then $\phi^{\mu}$ is one-one by 4.10.3. By 4.3 and 4.6, $\phi^{\mu+1}(f)=\left(\phi^{\mu}\right)^{1}(f)=\phi^{\mu} \circ f$ for each $f \in w^{\mu+1}(X)$, and hence $\operatorname{dom}\left(\phi^{\mu+1}(f)\right)=\operatorname{dom}(f)$ for each $f \in w^{\mu+1}(X)$. It is then seen from 2.4 and 4.4 that $\operatorname{dom}\left(\phi^{\mu}(f)\right)=\operatorname{dom}(f)$ for each $f \in w^{\mu}(X) \backslash \mathcal{u}^{*}$ if $\mu>0$. Q.e.d.

Proof of 4.10.5. By 4.3 and 4.6,

$$
\operatorname{im}\left(\phi^{\mu+1}(f)\right)=\operatorname{im}\left(\left(\phi^{\mu}\right)^{1}(f)\right)=\operatorname{im}\left(\phi^{\mu} \circ f\right)
$$

for each $f \in w^{\mu+1}(X)$. Q.e.d.

Proof of 4.10.6. We proceed by induction on $\mu$.

Consider $\mu=0$. For each $x \in w^{\circ}(X)=X$,

$$
\Lambda^{0}\left(\phi^{0}(x)\right)=\{[0, \phi(x)]\}=\phi \circ\{[0, x]\}=\phi \circ\left(\Lambda^{0}(x)\right)=\phi^{1}\left(\Lambda^{0}(x)\right)
$$

by $4.1,3.1$, and 4.6 .

Consider $\mu=1$. For each $f \in w^{1}(X)$,

$$
\Lambda^{1}\left(\phi^{1}(f)\right)=\phi^{1}(f)=\phi^{1}\left(\Lambda^{1}(f)\right)
$$

by 3.2 . 
Consider $\mu>1$ not a limit ordinal. Consider $f \in w^{\mu}(X)$. We have the following string of equations. [Equations (1) and (6) follow from $3.7 ;(2)$ follows from 4.10.5; (3) follows from the induction hypothesis; (4) and (8) follow from 4.6; (5) and (7) are immediate.]

$$
\begin{aligned}
& \operatorname{im}\left(\Lambda^{\mu}\left(\phi^{\mu}(f)\right)\right) \\
& =\bigcup_{\alpha \in \operatorname{dom}\left(\phi^{\mu}(f)\right)} \operatorname{im}\left(\Lambda^{\mu-1}\left(\phi^{\mu}(f)(\alpha)\right)\right) \\
& =\underset{\beta \in \operatorname{dom}(\gamma)}{\bigcup} \operatorname{im}\left(\Lambda^{\mu-1}\left(\phi^{\mu-1}(f(\beta))\right)\right) \\
& =\bigcup_{\beta \in \operatorname{dom}(\gamma)} \operatorname{im}\left(\phi^{1}\left(\Lambda^{\mu-1}(f(\beta))\right)\right) \\
& =\bigcup_{\beta \in \operatorname{dom}(\gamma)} \operatorname{im}\left(\phi \circ\left(\Lambda^{\mu-1}(f(\beta))\right)\right) \\
& =\operatorname{im}\left(\phi \mid \bigcup_{\beta \in \operatorname{dom}(\gamma)} \operatorname{im}\left(\Lambda^{\mu-1}(f(\beta))\right)\right) \\
& =\operatorname{im}\left(\phi \mid \operatorname{im}\left(\Lambda^{\mu}(f)\right)\right) \\
& =\operatorname{im}\left(\phi \circ\left(\Lambda^{\mu}(f)\right)\right) \\
& =\operatorname{im}\left(\phi^{1}\left(\Lambda^{\mu}(f)\right)\right) \text {. }
\end{aligned}
$$

Let

$$
\begin{aligned}
A & =\operatorname{im}\left(\Lambda^{\mu}\left(\phi^{\mu}(f)\right)\right)=\operatorname{im}\left(\phi^{1}\left(\Lambda^{\mu}(f)\right)\right), \\
R_{1} & =\left\{\left[\Lambda^{\mu}\left(\phi^{\mu}(f)\right)(\alpha), \Lambda^{\mu}\left(\phi^{\mu}(f)\right)(\beta)\right] \mid \alpha \leqq \beta \in \operatorname{dom}\left(\Lambda^{\mu}\left(\phi^{\mu}(f)\right)\right)\right\}, \\
R_{2} & =\left\{\left[\phi^{1}\left(\Lambda^{\mu}(f)\right)(\alpha), \phi^{1}\left(\Lambda^{\mu}(f)\right)(\beta)\right] \mid \alpha \leqq \beta \in \operatorname{dom}\left(\phi^{1}\left(\Lambda^{\mu}(f)\right)\right)\right\} .
\end{aligned}
$$

Then $R_{1} \cup R_{2} \subset A \times A$, and each of $R_{1}$ and $R_{2}$ reflexively well-orders $A$. To prove $\Lambda^{\mu}\left(\phi^{\mu}(f)\right)=\phi^{1}\left(\Lambda^{\mu}(f)\right)$ it suffices to prove $R_{1} \subset R_{2}$. Considering the righthand members of (1) and (2), we may make definitions (a) and (b) below.

(a) For each $x \in A$ let $\alpha_{x}$ be the smallest $\alpha \in \operatorname{dom}\left(\phi^{\mu}(f)\right)$ for which $x \in \operatorname{im}\left(\Lambda^{\mu-1}\left(\phi^{\mu}(f)(\alpha)\right)\right)$.

(b) For each $x \in A$ let $\beta_{x}$ be the smallest $\beta \in \operatorname{dom}(f)$ for which $x \in \operatorname{im}\left(\Lambda^{\mu-1}\left(\phi^{\mu-1}(f(\beta))\right)\right)$.

Considering (a), we may make definition (c) below.

(c) For each $x \in A$ let $\gamma_{x}$ be the smallest $\gamma \in \operatorname{dom}\left(\Lambda^{\mu-1}\left(\phi^{\mu}(f)\left(\alpha_{x}\right)\right)\right)$ for which $x=\Lambda^{\mu-1}\left(\phi^{\mu}(f)\left(\alpha_{x}\right)\right)(\gamma)$.

One may prove (d) and (e) below from (a), (b), 4.2, and 4.3.

(d) For each $x \in A, \phi^{\mu}(f)\left(\alpha_{x}\right)=\phi^{\mu-1}\left(f\left(\beta_{x}\right)\right)$.

(e) For all $x, y \in A, \alpha_{x}<\alpha_{y}$ if and only if $\beta_{x}<\beta_{y}$, and $\alpha_{x}=\alpha_{y}$ if and only if $\beta_{x}=\beta_{y}$.

By (c) and (d),

(f) For each $x \in A, \gamma_{x}$ is the smallest $\gamma \in \operatorname{dom}\left(\Lambda^{\mu-1}\left(\phi^{\mu-1}\left(f\left(\beta_{x}\right)\right)\right)\right)$ for which $x=\Lambda^{\mu-1}\left(\phi^{\mu-1}\left(f\left(\beta_{x}\right)\right)\right)(\gamma)$. 
By the induction hypothesis,

(g) For each $\beta \in \operatorname{dom}(f), \Lambda^{\mu-1}\left(\phi^{\mu-1}(f(\beta))\right)=\phi^{1}\left(\Lambda^{\mu-1}(f(\beta))\right)$.

By (g) and (f),

(h) For each $x \in A, \gamma_{x}$ is the smallest $\gamma \in \operatorname{dom}\left(\phi^{1}\left(\Lambda^{\mu-1}\left(f\left(\beta_{x}\right)\right)\right)\right)$ for which $x=\phi^{1}\left(\Lambda^{\mu-1}\left(f\left(\beta_{x}\right)\right)\right)(\gamma)$.

By $(\mathrm{g}),(\mathrm{b})$, and 4.6 ,

(i) For each $x \in A, \beta_{x}$ is the smallest $\beta \in \operatorname{dom}(f)$ for which $x \in \operatorname{im}\left(\phi^{1}\left(\Lambda^{\mu-1}(f(\beta))\right)\right)=\operatorname{im}\left(\phi \circ\left(\Lambda^{\mu-1}(f(\beta))\right)\right)$.

Considering $(\mathrm{h})$ and 4.6, we may make definition (j) below.

(j) For each $x \in A$ let $\delta_{x}$ be the smallest $\delta \in \operatorname{dom}\left(\Lambda^{\mu-1}\left(f\left(\beta_{x}\right)\right)\right)$ for which $x=\phi\left(\Lambda^{\mu-1}\left(f\left(\beta^{\mu}\right)\right)(\delta)\right)$.

By $(\mathrm{h}),(\mathrm{j})$, and 4.2 ,

(k) For each $x \in A, \gamma_{x} \leqq \gamma_{y}$ if and only if $\delta_{x} \leqq \delta_{y}$.

By (i) and (j),

(l) For each $x \in A, \Lambda^{\mu-1}\left(f\left(\beta_{x}\right)\right)\left(\delta_{x}\right) \notin \operatorname{im}\left(\Lambda^{\mu-1}(f(\beta))\right)$ for each $\beta<\beta_{x}$.

By (j),

(m) For each $x \in A, \Lambda^{\mu-1}\left(f\left(\beta_{x}\right)\right)(\delta) \neq \Lambda^{\mu-1}\left(f\left(\beta_{x}\right)\right)\left(\delta_{x}\right)$ for each $\delta<\delta_{x}$.

Let $S_{f}$ be given as in 3.3. Then, by 3.3, (1) and $(\mathrm{m})$,

(n) For all $x \in A$ and $\beta \in \operatorname{dom}(f)$ and $\delta \in \operatorname{dom}\left(\Lambda^{\mu-1}(f(\beta))\right)$,

$$
\Lambda^{\mu-1}(f(\beta))(\delta) S_{f} \Lambda^{\mu-1}\left(f\left(\beta_{x}\right)\right)\left(\delta_{x}\right)
$$

if and only if either $\beta<\beta_{x}$ or both $\beta=\beta_{x}$ and $\delta \leqq \delta_{x}$.

By (i), (j), (n), and 4.3,

(o) For all $x \in A$ and $t \in \operatorname{dom}\left(S_{f}\right)$ such that

$$
\begin{aligned}
& t S_{f} \Lambda^{\mu-1}\left(f\left(\beta_{x}\right)\right)\left(\delta_{x}\right), \\
& \phi(t) \neq \phi\left(\Lambda^{\mu-1}\left(f\left(\beta_{x}\right)\right)\left(\delta_{x}\right)\right) .
\end{aligned}
$$

Now consider $z, w \in A$ such that $z R_{1} w$. By (a), (c), and 3.3, either $\alpha_{z}<\alpha_{w}$ or both $\alpha_{z}=\alpha_{w}$ and $\gamma_{s} \leqq \gamma_{w}$. Hence, by (e) and (k), either $\beta_{z} \leqq \beta_{w}$ or both $\beta_{z}=\beta_{w}$ and $\delta_{z} \leqq \delta_{w}$. Hence, by $(n)$,

$$
\Lambda^{\mu-1}\left(f\left(\beta_{z}\right)\right)\left(\delta_{z}\right) S_{f} \Lambda^{\mu-1}\left(f\left(\beta_{w}\right)\right)\left(\delta_{w}\right) .
$$

Hence, by (o), 4.3, and 4.2,

$$
\phi\left(\Lambda^{\mu-1}\left(f\left(\beta_{z}\right)\right)\left(\delta_{z}\right)\right) R_{2} \phi\left(\Lambda^{\mu-1}\left(f\left(\beta_{w}\right)\right)\left(\delta_{w}\right)\right) .
$$

Hence, by (j), $z R_{2} w$. Thus $R_{1} \subset R_{2}$, and $\Lambda^{\mu}\left(\phi^{\mu}(f)\right)=\phi^{1}\left(\Lambda^{\mu}(f)\right)$.

Consider the case in which $\mu$ is a limit ordinal. Consider $f \in w^{\mu}(X)$. By 2.4 there is a first ordinal $\lambda<\mu$ for which $f \in w^{\lambda}(X)$, say $\lambda=\alpha$. By 4.8, $\alpha$ is the first ordinal $\lambda<\mu$ for which $\phi^{\mu}(f) \in w^{\lambda}(Y)$. By 4.7, $\phi^{\mu}(f)=\phi^{\alpha}(f)$. By 3.6 and the induction hypothesis,

$$
\begin{aligned}
\Lambda^{\mu}\left(\phi^{\mu}(f)\right) & =\Lambda^{\alpha}\left(\phi^{\mu}(f)\right)=\Lambda^{\alpha}\left(\phi^{\alpha}(f)\right) \\
& =\phi^{1}\left(\Lambda^{\alpha}(f)\right)=\phi^{1}\left(\Lambda^{\mu}(f)\right) .
\end{aligned}
$$


The induction is complete. Q.e.d.

The following theorem may be proved by induction. The details will be omitted.

4.11. Theorem. Suppose $\psi$ is a function whose domain is a set, suppose $\phi \subset \psi$, and suppose $\mu$ is an ordinal number. Suppose either $\mu$ is finite or $\operatorname{dom}(\psi)$ $\subset \mathcal{u}^{*}$. Then $\phi^{\mu} \subset \psi^{\mu}$.

Now suppose $\psi$ is a function (whose domain is not necessarily a set), suppose $\mu$ is an ordinal number, and suppose $\operatorname{dom}(\psi) \subset \mathcal{u}^{*}$ if $\mu$ is infinite. If $\psi$ is not a set (i.e., if $\operatorname{dom}(\psi)$ is not a set), we define

$$
\psi^{\mu}=\bigcup_{\phi \in \mathcal{P}(\psi)} \phi^{\mu}
$$

this equation holds by 4.11 if $\psi$ is a set. Then $\psi^{\mu}$ is a function, for, by 4.11 , if $\phi_{1}, \phi_{2} \in \mathcal{P}(\psi)$, then $\phi_{1}^{\mu} \cup \phi_{2}^{\mu} \subset\left(\phi_{1} \cup \phi_{2}\right)^{\mu}$ is a function. It may be proved from 4.5 that $\psi^{\mu}$ is a function from $W^{\mu}(\operatorname{dom}(\psi))$ to $W^{\mu}(\operatorname{im}(\psi))$. Obvious generalizations of 4.5-4.11 may be proved in a routine fashion, and 4.1-4.4 may be rephrased and generalized to theorems.

Suppose $\mu$ is an ordinal number. Let $\mathfrak{u}_{1}=\mathfrak{u}$ if $\mu$ is finite, and let $\mathfrak{u}_{1}=\mathfrak{u}^{*}$ if $\mu$ is infinite. For brevity we will operate in the meta-theory in the remainder of this paragraph. There is a correspondence which assigns $w^{\mu}(X)$ to each set $X \subset \mathfrak{U}_{1}$, and there is a correspondence which assigns the map $\phi^{\mu}: w^{\mu}(X)$ $\rightarrow \mathscr{W}^{\mu}(Y)$ to each map $\phi: X \rightarrow Y$ with $X \cup Y \in \mathcal{P}\left(\mathfrak{U}_{1}\right)$. In the parlance of categories and functors [2], by 4.10.1-4.10.3 these two correspondences constitute a covariant functor from the category of subsets of $\mathfrak{u}_{1}$ and their maps to the category of sets and their maps. This functor conforms with $\Lambda^{\mu}$ in a nice way (cf. 4.10.6). If $\mathfrak{u}_{1}$ is replaced by $\mathcal{u}^{*}$, by 3.9 and 4.7 the superscripts on " $\Lambda$ " and " $\phi$ " could be dropped for ordinary purposes.

5. Free elements. We define $\mu$-free element for each ordinal number $\mu$ by transfinite induction as follows (5.1-5.4).

5.1. An element $x$ is 0 -free if and only if $x \in \mathcal{u}^{*}$.

5.2. An element $f$ is 1 -free if and only if $f \in W\left(\mathcal{U}^{*}\right)$.

5.3. An element $f$ is $(\mu+1)$-free if and only if $f \in w^{\mu+1}\left(\mathcal{U}^{*}\right), f(\alpha)$ is $\mu$-free for each $\alpha \in \operatorname{dom}(f)$, and

$$
\left[\operatorname{im}\left(\Lambda^{\mu}(f(\alpha))\right)\right] \cap\left[\operatorname{im}\left(\Lambda^{\mu}(f(\beta))\right)\right]=0
$$

for all $\alpha, \beta \in \operatorname{dom}(f)$ with $\alpha \neq \beta$. [This holds for $\mu=0$ by 5.1 and 5.2.]

5.4. Suppose $\mu$ is a limit ordinal number. An element $f$ is $\mu$-free if and only if $f \in w^{\mu}\left(\mathcal{U}^{*}\right)$ and, where $\gamma$ is the first $\lambda<\mu$ for which $f \in w^{\lambda}\left(\mathcal{u}^{*}\right)$ (cf. 2.4), $f$ is $\gamma$-free.

5.5. Theorem. Suppose $\mu$ and $\nu$ are ordinal numbers, and suppose $f \in w^{\mu}\left(\mathcal{U}^{*}\right) \cap w^{\nu}\left(\mathcal{u}^{*}\right)$. Then $f$ is $\mu$-free if and only if $f$ is $\nu$-free. 
Proof. We proceed by induction on $\max (\mu, \nu)$. We may suppose $\mu<\nu$. Then $\nu>0$. If $\nu=1$, then $\mu=0$, and $f \in \mathcal{U}^{*} \cap \Re=0$, a contradiction. Hence we may suppose $\nu>1$. By 5.4 we may suppose neither $\mu$ nor $\nu$ is a limit ordinal. If $\mu=0$, it is easily seen that $\nu$ must be a limit ordinal contrary to supposition. Hence $\mu>0$. By 5.3, 3.8, and the induction hypothesis, $f$ if $\mu$-free if and only if $f$ is $\nu$-free. Q.e.d.

For each element $x, x$ will be said to be free if and only if $x$ is $\mu$-free for some ordinal number $\mu$. Then, by 5.5 , for each ordinal number $\nu$ and each $f \in W^{\nu}\left(\mathcal{U}^{*}\right), f$ is free if and only if $f$ is $\nu$-free.

The following theorem may be proved by induction using 5.1-5.4, 4.1, 4.4, 4.10.4, and 4.10.5. The details will be omitted.

5.6. Theorem. Suppose $X \subset \mathcal{U}^{*}$ and $Y \subset \mathcal{U}^{*}$ are sets and $\phi$ a one-one function with domain $X$ and image $Y$. Then for each ordinal number $\mu$ and each $f \in w^{\mu}(X), f$ is free if and only if $\phi^{\mu}(f)$ is free.

One of the main goals of the rest of this section is to prove 5.21. By 5.20, 5.21 holds "uniformly." The procedure will be somewhat lengthy, but rather straightforward.

The next lemma and its corollary are purely technical.

5.7. Lemma. Suppose $x, y \in \mathcal{U}$. Then $[x, y] \in \mathfrak{N}$ if and only if $x=y=1$. Moreover, $[1,1]=\{[0,0]\}$.

Proof. Suppose

$$
\{\{x\},\{x, y\}\}=[x, y] \in \Re .
$$

There are $u, v \in \mathcal{u}$ and ordinal numbers $\alpha$ and $\beta$ such that

$$
\{x\}=[\alpha, u]=\{\{\alpha\},\{\alpha, u\}\}, \quad\{x, y\}=[\beta, v]=\{\{\beta\},\{\beta, v\}\} .
$$

It follows that $x=\{\alpha\}=\{\alpha, u\}$ and $\alpha=u$. Moreover, $\{\alpha\}=x=\{\beta\}$ or $\{\alpha\}=x=\{\beta, v\}$. Hence $\alpha=\beta$. Since $\operatorname{dom}([x, y])=\{\alpha, \beta\}=\{\alpha\}$ is an ordinal number with cardinal 1 , we have $x=\{\alpha\}=1=\{0\}$, and $\beta=\alpha=0$. Since $[x, y]=\{[0, u],[0, v]\}$ is a function, we have $v=u=\alpha=0$. Hence $y=\{\beta\}$ $=\{0\}=x=1$ or $y=\{\beta, v\}=\{0\}=x=1$. Moreover, since

$$
[z, z]=\{\{z\},\{z, z\}\}=\{\{z\}\}
$$

for each $s \in \mathcal{u}$, we have

$$
[1,1]=\{\{1\}\}=\{\{\{0\}\}\}=\{[0,0]\} .
$$

Q.e.d.

5.8. Corollary. For each function $f$ and each $x \in \mathcal{u},[f, x] \in \mathcal{u}^{*}$.

Proof. Since ordered pairs are nonvoid sets, 0 is not an ordered pair. Since $0 \in 1,1$ is not a function. Now apply 5.7. Q.e.d. 
If $X$ is a set, an $X$-tuple is any function with domain $X$. We now define

$T_{\mu}=\{x \mid x$ is a function such that $\operatorname{dom}(x)$ is a finite ordinal number and $\operatorname{im}(x) \subset \mu\}$

for each ordinal number $\mu$, and we define

$$
\mathfrak{J}=\bigcup_{\mu \in \mathcal{O}} T_{\mu}
$$

Thus $x \in T_{\mu}$ if and only if $x$ is a $\lambda$-tuple of ordinal numbers $<\mu$ for some finite ordinal $\lambda$. For each ordinal number $\mu, 0 \in T_{\mu}$ since 0 is a 0 -tuple.

Suppose $X$ is a set. We define $\Phi_{X}^{\mu}(f)$ for each ordinal number $\mu$ and each $f \in w^{\mu}(X)$ by induction as follows (5.9-5.11) so that $\Phi_{X}^{\mu}(f)$ is a function with image $\operatorname{im}\left(\Lambda^{\mu}(f)\right)$ and with domain $\subset J \times X$.

5.9. If $f \in w^{\circ}(X)=X$, let

Then

$$
\Phi_{\mathbf{X}}^{0}(f)=\{[[0, f], f]\} .
$$

$$
\begin{aligned}
\operatorname{dom}\left(\Phi_{X}^{\mu}(f)\right) & =\{[0, f]\} \subset \Im \times X, \\
\operatorname{im}\left(\Phi_{X}^{\mu}(f)\right) & =\{f\}=\operatorname{im}\left(\Lambda^{0}(f)\right) .
\end{aligned}
$$

5.10. Suppose $\mu>0$ is a nonlimit ordinal number. Consider $f \in w^{\mu}(X)$. Let

$$
\begin{array}{r}
\Phi_{X}^{\mu}(f)=\left\{\left[[t \cup\{[\operatorname{dom}(t), \alpha]\}, x], \Phi_{X}^{\mu-1}(f(\alpha))([t, x])\right] \mid\right. \\
\left.\alpha \in \operatorname{dom}(f) ;[t, x] \in \operatorname{dom}\left(\Phi_{X}^{\mu-1}(f(\alpha))\right)\right\} .
\end{array}
$$

By the induction hypothesis and 3.7, $\Phi_{X}^{\mu}(f)$ is a function with image

$$
\underset{\alpha \in \operatorname{dom}(f)}{\bigcup} \operatorname{im}\left(\Phi_{X}^{\mu-1}(f(\alpha))\right)=\underset{\alpha \in \operatorname{dom}(f)}{U} \operatorname{im}\left(\Lambda^{\mu-1}(f(\alpha))\right)=\operatorname{im}\left(\Lambda^{\mu}(f)\right)
$$

and with domain $\subset \Im \times X$.

5.11. Suppose $\mu$ is a limit ordinal number. Then let $\Phi_{X}^{\mu}(f)=\Phi_{X}^{\gamma}(f)$ where $\gamma$ is the first $\lambda<\mu$ such that $f \in w^{\lambda}(X)$ (cf. 2.4). Then $\Phi_{X}^{\mu}(f)$ is a function with image $\operatorname{im}\left(\Lambda^{\gamma}(f)\right)=\operatorname{im}\left(\Lambda^{\mu}(f)\right)$ and domain $C J \times X$ by the induction hypothesis and 3.9.

The following theorem may be proved by induction. The details will be omitted.

5.12. Theorem. Suppose $\mu$ is an ordinal number and $X$ and $Y$ are sets. Suppose either $\mu$ is finite or $X \cup Y \subset \mathcal{U}^{*}$. Suppose $f \in w^{\mu}(X) \cap w^{\mu}(Y)$. Then $\Phi_{\bar{X}}^{\mu}(f)=\Phi_{Y}^{\mu}(f)$.

Suppose $\mu$ is an ordinal number. Let $\mathfrak{u}_{1}=\mathfrak{u}$ if $\mu$ is finite, and let $\mathfrak{u}_{1}=\mathfrak{u}^{*}$ if $\mu$ is infinite. For each set $X \subset \mathcal{u}_{1}$,

$$
\Phi_{X}^{\mu}=\left\{\left[f, \Phi_{X}^{\mu}(f)\right] \mid f \in W^{\mu}(X)\right\}
$$


is a function with domain $W^{\mu}(X)$. By 5.12,

$$
\Phi^{\mu}=\bigcup_{x \in \mathcal{P}\left(\mathcal{U}_{1}\right)} \Phi_{x}^{\mu}
$$

is a function with domain $w^{\mu}\left(\mathcal{u}_{1}\right)$, and, for each set $X \subset \mathfrak{U}_{1}$ and each $f \in W^{\mu}(X), \Phi^{\mu}(f)=\Phi_{X}^{\mu}(f)$.

5.13. TheоRем. Suppose $\mu$ and $\nu$ are ordinal numbers and $f \in w^{\mu}\left(\mathcal{U}^{*}\right)$ $\cap w^{\nu}\left(\mathcal{U}^{*}\right)$. Then $\Phi^{\mu}(f)=\Phi^{\prime}(f)$.

Proof. We proceed by induction on $\max (\mu, \nu)$. We may suppose $\mu<\nu$. Then $\nu>0$. If $\nu=1$, then $\mu=0$, and $f \in \mathcal{U}^{*} \cap \Re=0$, a contradiction. Hence we may suppose $\nu>1$. By 5.11 we may suppose neither $\mu$ nor $\nu$ is a limit ordinal. If $\mu=0$, it is easily seen that $\nu$ must be a limit ordinal contrary to supposition. Hence $\mu>0$. Now, by 5.10 and the induction hypothesis, $\Phi^{\mu}(f)=\Phi^{\nu}(f)$. Q.e.d.

5.14. Theorem. Suppose $\mu$ is an ordinal number and $f \in w^{\mu}(\mathcal{u})$, and sup-

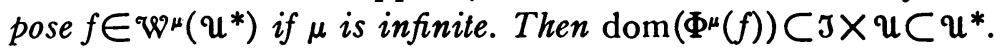

Proof. Apply 5.8. Q.e.d.

Suppose $X$ is a set. We define $\Gamma_{X}^{\mu}(f)$ for each finite ordinal number $\mu$ and each $f \in W^{\mu}(X)$, and also for each infinite ordinal number $\mu$ and each $f \in W^{\mu}(X)$ if $X \subset \mathcal{U}^{*}$, by induction as follows (5.15-5.17) so that (A), (B), and (C) below hold.

(A) For each $f \in w^{\mu}(X), \Gamma_{X}^{\mu}(f) \in w^{\mu}\left(\operatorname{dom}\left(\Phi^{\mu}(f)\right)\right)$.

(B) The function $\Gamma_{X}^{\mu}=\left\{\left[g, \Gamma_{X}^{\mu}(g)\right] \mid g \in W^{\mu}(X)\right\}$ is one-one.

(C) If $X \subset \mathcal{U}^{*}$, then for each $f \in W^{\mu}(X)$ and each ordinal number $\lambda, \Gamma_{X}^{\mu}(f)$ $\in w^{\lambda}\left(\mathcal{U}^{*}\right)$ if and only if $f \in W^{\lambda}(X)$.

5.15. If $f \in W^{0}(X)=X$, let $\Gamma_{X}^{0}(f)=[0, f]$.

In 5.15, for each $f \in W^{0}(X), \Gamma_{X}^{0}(f)=[0, f] \in \operatorname{dom}\left(\Phi^{0}(X)\right)$ by 5.9. Thus (A) holds. Trivially, (B) holds. Suppose $X \subset \mathfrak{u}^{*}$. Then for each $f \in w^{0}(X), f \in \mathcal{u}^{*}$ and $[0, f] \in \mathcal{U}^{*}$ by 5.7. Hence (cf. 2.1-2.4) for each ordinal number $\lambda, f$ $\in W^{\lambda}(X)$ if and only if $\lambda$ is a limit ordinal, and $[0, f] \in w^{\lambda}\left(\mathcal{u}^{*}\right)$ if and only if $\lambda$ is a limit ordinal. Thus (C) holds.

5.16. Suppose $\mu>0$ is a nonlimit ordinal. Consider $f \in W^{\mu}(X)$. Consider $\alpha \in \operatorname{dom}(f)$. By the induction hypothesis,

$$
\begin{aligned}
\Gamma_{X}^{\mu-1}(f(\alpha)) & \in W^{\mu-1}\left(\operatorname{dom}\left(\Phi^{\mu-1}(f(\alpha))\right)\right), \\
\operatorname{im}\left(\Lambda^{\mu-1}\left(\Gamma_{X}^{\mu-1}(f(\alpha))\right)\right) & \subset \operatorname{dom}\left(\Phi^{\mu-1}(f(\alpha))\right) \subset \Im \times X .
\end{aligned}
$$

Let

$$
\xi_{\alpha}=\left\{[[t, x],[t \cup\{[\operatorname{dom}(t), \alpha]\}, x]] \mid[t, x] \in \operatorname{im}\left(\Lambda^{\mu-1}\left(\Gamma_{X}^{\mu-1}(f(\alpha))\right)\right)\right\} .
$$

Then $\xi_{\alpha}$ is a one-one function such that (cf. 5.8) 


$$
\begin{aligned}
\operatorname{dom}\left(\xi_{\alpha}\right) & =\operatorname{im}\left(\Lambda^{\mu-1}\left(\Gamma_{X}^{\mu-1}(f(\alpha))\right)\right) \subset \operatorname{dom}\left(\Phi^{\mu-1}(f(\alpha))\right) \\
\operatorname{im}\left(\xi_{\alpha}\right) & \subset \operatorname{dom}\left(\Phi^{\mu}(f)\right)
\end{aligned}
$$

Let

$$
f_{\alpha}=\xi_{\alpha}^{\mu-1}\left(\Gamma_{X}^{\mu-1}(f(\alpha))\right)
$$

Then

$$
f_{\alpha} \in W^{\mu-1}\left(\operatorname{dom}\left(\Phi^{\mu}(f)\right)\right)
$$

Let

$$
\Gamma_{X}^{\mu}(f)=\left\{\left[\beta, f_{\beta}\right] \mid \beta \in \operatorname{dom}(f)\right\} .
$$

In 5.16 we will use the induction hypothesis to show that (A) holds. For each $\alpha \in \operatorname{dom}(f)$ observe from $5.16,4.10 .6,4.6$, and 5.16 respectively that

$$
\begin{aligned}
\operatorname{im}\left(\Lambda^{\mu-1}\left(\Gamma_{X}^{\mu}(f)(\alpha)\right)\right. & =\operatorname{im}\left(\Lambda^{\mu-1}\left(\xi_{\alpha}^{\mu-1}\left(\Gamma_{X}^{\mu-1}(f(\alpha))\right)\right)\right) \\
& =\operatorname{im}\left(\xi_{\alpha}^{1}\left(\Lambda^{\mu-1}\left(\Gamma_{X}^{\mu-1}(f(\alpha))\right)\right)\right) \\
& =\operatorname{im}\left(\xi_{\alpha} \circ\left(\Lambda^{\mu-1}\left(\Gamma_{X}^{\mu-1}(f(\alpha))\right)\right)\right) \\
& =\operatorname{im}\left(\xi_{\alpha}\right) .
\end{aligned}
$$

Now consider $\beta<\gamma \in \operatorname{dom}(f)$. If either $\xi_{\alpha}$ or $\xi_{\gamma}$ is nonvoid, then, by 5.16,

$$
\operatorname{im}\left(\Lambda^{\mu-1}\left(\Gamma_{X}^{\mu}(f)(\beta)\right)\right)=\operatorname{im}\left(\xi_{\beta}\right) \neq \operatorname{im}\left(\xi_{\gamma}\right)=\operatorname{im}\left(\Lambda^{\mu-1}\left(\Gamma_{X}^{\mu}(f)(\gamma)\right)\right),
$$

and $\Gamma_{X}^{\mu}(f)(\beta) \neq \Gamma_{X}^{\mu}(f)(\gamma)$. If $\xi_{\beta}=\xi_{\gamma}=0$, then $\xi_{\gamma}^{\mu-1}=\xi_{\beta}^{\mu-1}$ is one-one by 4.10 .4 , and (by 5.16 and the fact that $\Gamma_{X}^{\mu-1}(f(\beta)) \neq \Gamma_{X}^{\mu-1}(f(\gamma))$ by the induction hypothesis (cf. (B)))

$$
\Gamma_{X}^{\mu}(f)(\beta)=\xi_{\beta}^{\mu-1}\left(\Gamma_{X}^{\mu-1}(f(\beta))\right) \neq \xi_{\gamma}^{\mu-1}\left(\Gamma_{X}^{\mu-1}(f(\gamma))\right)=\Gamma_{X}^{\mu}(f)(\gamma) .
$$

Thus $\Gamma_{X}^{\mu}(f)$ is one-one, and

$$
\left.\Gamma_{X}^{\mu}(f) \in w_{\left(w^{\mu-1}\right.}\left(\operatorname{dom}\left(\Phi^{\mu}(f)\right)\right)\right)
$$

Thus (A) holds.

Next we will ase the induction hypothesis to show that $(B)$ holds. Consider $f, g \in w^{\mu}(X)$ such that $f \neq g$. We must show that $\Gamma_{X}^{\mu}(f) \neq \Gamma_{X}^{\mu}(g)$. If $\operatorname{dom}(f) \neq \operatorname{dom}(g)$, then

$$
\operatorname{dom}\left(\Gamma_{X}^{\mu}(f)\right)=\operatorname{dom}(f) \neq \operatorname{dom}(g)=\operatorname{dom}\left(\Gamma_{X}^{\mu}(g)\right),
$$

and $\Gamma_{X}^{\mu}(f) \neq \Gamma_{X}^{\mu}(g)$. Suppose $\operatorname{dom}(f)=\operatorname{dom}(g)$. Then $f(\beta) \neq g(\beta)$ for some $\beta \in \operatorname{dom}(f)$. Consider such a $\beta$. Let $\xi_{\beta}$ be defined as in 5.16 , and let $\xi_{\beta}^{\prime}$ be defined similarly with $f$ replaced by $g$. If $\xi_{\beta} \neq \xi_{\beta}^{\prime}$, then $\operatorname{im}\left(\xi_{\beta}\right) \neq \operatorname{im}\left(\xi_{\beta}^{\prime}\right)$ (cf. 
5.16), and (cf. the preceding paragraph)

$$
\operatorname{im}\left(\Lambda^{\mu-1}\left(\Gamma_{X}^{\mu}(f)(\beta)\right)\right)=\operatorname{im}\left(\xi_{\beta}\right) \neq \operatorname{im}\left(\xi_{\beta}^{\prime}\right)=\operatorname{im}\left(\Lambda^{\mu-1}\left(\Gamma_{X}^{\mu}(g)(\beta)\right)\right),
$$

and hence $\Gamma_{X}^{\mu}(f)(\beta) \neq \Gamma_{X}^{\mu}(g)(\beta)$. If $\xi_{\beta}=\xi_{\beta}^{\prime}$, then, by $5.16,4.10 .4$ (since $\xi_{\beta}$ is one-one), and the fact that $\Gamma_{X}^{\mu-1}(f(\beta)) \neq \Gamma_{X}^{\mu-1}(g(\beta))$ by the induction hypothesis,

$$
\Gamma_{X}^{\mu}(f)(\beta)=\xi_{\beta}^{\mu-1}\left(\Gamma_{X}^{\mu-1}(f(\beta))\right) \neq \dot{\xi}_{\beta}^{\mu-1}\left(\Gamma_{X}^{\mu-1}(g(\beta))\right)=\Gamma_{X}^{\mu}(g)(\beta) .
$$

Thus $\Gamma_{X}^{\mu}(f)(\beta) \neq \Gamma_{X}^{\mu}(g)(\beta)$ if $f(\beta) \neq g(\beta)$. Thus $\Gamma_{X}^{\mu}(f) \neq \Gamma_{X}^{\mu}(g)$. Thus (B) holds.

Next we will use the induction hypothesis to show that (C) holds. Consider $X \subset \mathcal{U}^{*}$ and $f \in w^{\mu}(X)$. For each $\alpha \in \operatorname{dom}(f)$ and each ordinal number $\lambda$; in 5.16,

$$
\operatorname{dom}\left(\xi_{\alpha}\right) \cup \operatorname{im}\left(\xi_{\alpha}\right) \subset \operatorname{dom}\left(\Phi^{\mu-1}(f(\alpha))\right) \cup \operatorname{dom}\left(\Phi^{\mu}(f)\right) \subset \mathcal{U}^{*}
$$

by 5.14 , and hence, by 4.8 (cf. also 2.8 ),

$$
\Gamma_{X}^{\mu}(f)(\alpha)=\xi_{\alpha}^{\mu-1}\left(\Gamma_{X}^{\mu-1}(f(\alpha))\right) \in w^{\lambda}\left(u^{*}\right)
$$

if and only if $\Gamma_{X}^{\mu-1}(f(\alpha)) \in W^{\lambda}\left(\mathcal{u}^{*}\right)$ and hence, by the induction hypothesis, if and only if $f(\alpha) \in w^{\lambda}\left(\mathcal{U}^{*}\right)$. Also, for each ordinal number $\lambda, f \in w^{\lambda+1}(X)$ if and only if $f(\alpha) \in w^{\lambda}(X)$ for each $\alpha \in \operatorname{dom}(f)$ (cf. 2.3), hence if and only if $\Gamma_{X}^{\mu}(f)(\alpha) \in W^{\lambda}\left(u^{*}\right)$ for each $\alpha \in \operatorname{dom}(f)=\operatorname{dom}\left(\Gamma_{X}^{\mu}(f)\right)$, and hence if and only if $\Gamma_{X}^{\mu}(f) \in w^{\lambda+1}\left(\mathcal{U}^{*}\right)$ (cf. 2.3 and 2.8). Since $f \in w^{\mu}(X) \subset \Re$ and $\Gamma_{X}^{\mu}(f) \in \Re$, we have $f \notin X=W^{0}(X)$ and $\Gamma_{X}^{\mu}(f) \notin \mathcal{U}^{*}=W^{0}\left(\mathcal{U}^{*}\right)$. Now by 2.4 (and 2.8) and the preceding part of this paragraph, if $\lambda$ is a limit ordinal, $f \in W^{\lambda}(X)$ if and only if $\Gamma_{X}^{\mu}(f) \in w^{\lambda}\left(\mathcal{u}^{*}\right)$. Thus $(C)$ holds.

Thus $\mathbf{5 . 1 6}$ preserves the induction hypothesis.

5.17. Suppose $\mu>0$ is a limit ordinal number. Consider $f \in w^{\mu}(X)$. Let $\gamma$ be the first ordinal $\lambda<\mu$ such that $f \in W^{\lambda}(X)$ (cf. 2.4). Let $\Gamma_{X}^{\mu}(f)=\Gamma_{X}^{\gamma}(f)$.

Then (A) holds by the induction hypothesis (which implies $\Gamma_{X}^{\gamma}(f)$ $\in W^{\gamma}\left(\operatorname{dom}\left(\Phi^{\gamma}(f)\right)\right)$ in 5.17), 5.13, and 2.4. Also, (C) holds by the induction hypothesis. To prove (B) consider $f, g \in w^{\mu}(X)$ with $\Gamma_{X}^{\mu}(f)=\Gamma_{X}^{\mu}(g)$. Let $\gamma$ be the first $\lambda<\mu$ for which $f \in W^{\lambda}(X)$, and let $\delta$ be the first $\lambda<\mu$ for which $g \in w^{\lambda}(X)$. Then, by $(C), \gamma$ is the first $\lambda<\mu$ for which $\Gamma_{X}^{\mu}(f) \in w^{\lambda}\left(\mathcal{u}_{0}\right)$, and $\delta$ is the first $\lambda<\mu$ for which $\Gamma_{X}^{\mu}(f)=\Gamma_{X}^{\mu}(g) \in w^{\lambda}\left(\mathcal{u}_{0}\right)$. Hence $\gamma=\delta$. Then $\Gamma_{X}^{\gamma}(f)$ $=\Gamma_{X}^{\mu}(f)=\Gamma_{X}^{\mu}(g)=\Gamma_{X}^{\gamma}(g)$, and hence $f=g$ by the induction hypothesis. Thus (B) is established.

The definition via 5.15-5.17 of $\Gamma_{X}^{\mu}(f)$ for all $\mu, X$, and $f$ as specified at the outset is now justified.

The following theorem may be proved from 2.8 and $5.15-5.17$ by induction. We omit the details.

5.18. Theorem. Suppose $\mu$ is an ordinal number and $X$ and $Y$ are sets, 
and suppose $f \in w^{\mu}(X) \cap w^{\mu}(Y)$. Suppose $\mu$ is finite or $X \cup Y \subset \mathcal{u}^{*}$. Then $\Gamma_{X}^{\mu}(f)=\Gamma_{Y}^{\mu}(f)$.

Suppose $\mu$ is an ordinal number. Let $\mathfrak{u}_{1}=\mathfrak{u}$ if $\mu$ is finite. and let $\mathfrak{u}_{1}=\mathcal{u}^{*}$ if $\mu$ is infinite. By 5.18 ,

$$
\Gamma^{\mu}=\bigcup_{x \in \mathcal{P}\left(\mathcal{U}_{1}\right)} \Gamma_{x}^{\mu}
$$

is a function with domain $w^{\mu}\left(\mathcal{u}_{1}\right)$, and for each set $X \subset \mathcal{u}_{1}$ and each $f \in w^{\mu}(X)$, $\Gamma^{\mu}(f)=\Gamma_{\mathbf{X}}^{\mu}(f)$.

To prove the following theorem proceed as in the proof of 5.13, but use 5.17 and 5.16 instead of 5.11 and 5.10. Use also 5.13. The details will be omitted.

5.19. Theorem. Suppose $\mu$ and $\nu$ are ordinal numbers and $f \in w^{\mu}\left(u^{*}\right)$ $\cap w^{\vee}\left(\mathcal{U}^{*}\right)$. Then $\Gamma^{\mu}(f)=\Gamma^{\nu}(f)$.

5.20. Theorem. Suppose $\mu$ is an ordinal number and $f \in \mathcal{w}^{\mu}(\mathcal{u})$, and suppose $f \in w^{\mu}\left(\mathcal{U}^{*}\right)$ if $\mu$ is infinite. Then 5.20.1-5.20.4 below hold.

5.20.1. $\operatorname{dom}\left(\Gamma^{\mu}(f)\right)=\operatorname{dom}(f)$ if $\mu>0$.

5.20.2. $\Gamma^{\mu}(f)$ is free.

5.20.3. $\Phi^{\mu}(f)$ is a function whose domain is $\operatorname{im}\left(\Lambda^{\mu}\left(\Gamma^{\mu}(f)\right)\right) \subset \mathcal{U}^{*}$ and whose image is $\operatorname{im}\left(\Lambda^{\mu}(f)\right)$.

5.20.4. $\left[\Phi^{\mu}(f)\right]^{\mu}\left(\Gamma^{\mu}(f)\right)=f$.

Proof of 5.20.1. Apply 5.16 and 5.17. Q.e.d.

Proof of 5.20.2. We proceed by induction. For $\mu=0$ apply 5.15, 5.7, and 5.1.

Consider $\bar{\mu}>0$ not a limit ordinal. Consider $\alpha \in \operatorname{dom}(f)=\operatorname{dom}\left(\Gamma^{\mu}(f)\right)$. In 5.16, $\operatorname{dom}\left(\xi_{\alpha}\right) \cup \operatorname{im}\left(\xi_{\alpha}\right) \subset \mathcal{U}^{*}$ by 5.14 , and $\xi_{\alpha}$ is one-one. Also, $\Gamma^{\mu-1}(f(\alpha))$ is free by the induction hypothesis. Hence (cf. 5.16)

$$
\Gamma^{\mu}(f)(\alpha)=\xi_{\alpha}^{\mu-1}\left(\Gamma^{\mu-1}(f(\alpha))\right)
$$

is free by 5.6. Also, as in the proof of (A) for the $\mu$ of 5.16,

$$
\operatorname{im}\left(\Lambda^{\mu-1}\left(\Gamma^{\mu}(f)(\alpha)\right)\right)=\operatorname{im}\left(\xi_{\alpha}\right) .
$$

In 5.16, if $\alpha<\beta \in \operatorname{dom}(f)$, then $\operatorname{im}\left(\xi_{\alpha}\right) \cap \operatorname{im}\left(\xi_{\beta}\right)=0$. Hence $\Gamma^{\mu}(f)$ is free by 5.3.

Consider $\mu$ a limit ordinal. Then $\Gamma^{\mu}(f)$ is free by $5.17,5.4$, and the induction hypothesis.

The induction is complete. Q.e.d.

Proof of 5.20.3. (First observe that $\operatorname{dom}\left(\Phi^{\mu}(f)\right) \subset \mathcal{U}^{*}$ by 5.14.) We proceed by induction. For $\mu=0,5.20 .3$ follows from 5.9, 5.15, and 3.1.

Consider $\mu>0$ not a limit ordinal. By 5.10, $\Phi_{X}^{\mu}(f)$ is a function. We have the following equations. [Equation (1) follows from 5.10 and the induction 
hypothesis; (2) and (5) follow from 5.16; (3) follows from 4.6; (4) follows from 4.10.6; (6) follows from 3.7.]

$$
\operatorname{dom}\left(\Phi^{\mu}(f)\right)
$$

$$
\begin{aligned}
& =\{[t \cup\{[\operatorname{dom}(t), \alpha]\}, x] \mid \alpha \in \operatorname{dom}(f) ; \\
& \left.[t, x] \in \operatorname{dom}\left(\Phi^{\mu-1}(f(\alpha))\right)=\operatorname{im}\left(\Lambda^{\mu-1}\left(\Gamma^{\mu-1}(f(\alpha))\right)\right)\right\} \\
& =\underset{\alpha \in \operatorname{dom}(\zeta)}{U} \operatorname{im}\left(\xi_{\alpha} \circ\left(\Lambda^{\mu-1}\left(\Gamma^{\mu-1}(f(\alpha))\right)\right)\right) \\
& =\underset{\alpha \in \operatorname{dom}(\zeta)}{U} \operatorname{im}\left(\xi_{\alpha}^{1}\left(\Lambda^{\mu-1}\left(\Gamma^{\mu-1}(f(\alpha))\right)\right)\right) \\
& =\bigcup_{\alpha \in \operatorname{dom}())} \operatorname{im}\left(\Lambda^{\mu-1}\left(\xi_{\alpha}^{\mu-1}\left(\Gamma^{\mu-1}(f(\alpha))\right)\right)\right) \\
& =\bigcup_{\alpha \in \operatorname{dom}())}^{U} \operatorname{im}\left(\Lambda^{\mu-1}\left(\Gamma^{\mu}(f)(\alpha)\right)\right) \\
& =\operatorname{im}\left(\Lambda^{\mu}\left(\Gamma^{\mu}(f)\right)\right) .
\end{aligned}
$$

By 5.10, the induction hypothesis, and 3.7,

$$
\begin{aligned}
\operatorname{im}\left(\Phi^{\mu}(f)\right) & =\bigcup_{\alpha \in \operatorname{dom}(\gamma)} \operatorname{im}\left(\Phi^{\mu-1}(f(\alpha))\right) \\
& =\bigcup_{\alpha \in \operatorname{dom}(\gamma)} \operatorname{im}\left(\Lambda^{\mu-1}(f(\alpha))\right) \\
& =\operatorname{im}\left(\Lambda^{\mu}(f)\right) .
\end{aligned}
$$

Now consider $\mu$ a limit ordinal. Then $\mathbf{5 . 2 0 . 3}$ follows from 5.11, the induction hypothesis, 5.17, and 3.9.

The induction is complete. Q.e.d.

Proof of 5.20.4. We proceed by induction. For $\mu=0$ apply 5.15, 5.9, and 4.1.

Consider $\mu>0$ not a limit ordinal. Consider $\alpha \in \operatorname{dom}\left(\Gamma^{\mu}(f)\right)=\operatorname{dom}(f)$. In the notation of 5.16 , by $5.10,5.16$, and 5.20 .3 ,

$$
\Phi^{\mu-1}(f(\alpha))=\Phi^{\mu}(f) \circ \xi_{\alpha} .
$$

Then, by $5.16,4.10 .1$, and the induction hypothesis,

$$
\begin{aligned}
{\left[\Phi^{\mu}(f)\right]^{\mu-1}\left(\Gamma^{\mu}(f)(\alpha)\right) } & =\left[\Phi^{\mu}(f)\right]^{\mu-1}\left(\xi_{\alpha}^{\mu-1}\left(\Gamma^{\mu-1}(f(\alpha))\right)\right) \\
& =\left[\Phi^{\mu}(f) \circ \xi_{\alpha}\right]^{\mu-1}\left(\Gamma^{\mu-1}(f(\alpha))\right) \\
& =\left[\Phi^{\mu-1}(f(\alpha))\right]^{\mu-1}\left(\Gamma^{\mu-1}(f(\alpha))\right)=f(\alpha) .
\end{aligned}
$$

It now follows easily from 4.2 and 4.3 (since $f(\beta) \neq f(\gamma)$ if $\beta<\gamma \in \operatorname{dom}(f)$ ) that

$$
\left[\Phi^{\mu}(f)\right]^{\mu}\left(\Gamma^{\mu}(f)\right)=\left[\Phi^{\mu}(f)\right]^{\mu-1} \circ\left[\Gamma^{\mu}(f)\right]=f .
$$

Consider $\mu$ a limit ordinal. Then 5.20 .4 follows from $5.17,5.11,4.7$, and the induction hypothesis. 
The induction is complete. Q.e.d.

In 5.20 take $Y=\operatorname{dom}\left(\Phi^{\mu}(f)\right), g=\Gamma^{\mu}(f)$, and $\phi=\Phi^{\mu}(f)$ to get the following corollary.

5.21. Corollary. Suppose $\mu$ is an ordinal number and $f \in W^{\mu}(\mathcal{U})$, and suppose $f \in W^{\mu}\left(\mathcal{U}^{*}\right)$ if $\mu$ is infinite. Then there are a set $Y \subset \mathcal{U}^{*}$, a free element $g \in W^{\mu}(Y)$, and a function $\phi$ from $Y$ onto $\operatorname{im}\left(\Lambda^{\mu}(f)\right)$ such that $f=\phi^{\mu}(g)$.

As remarked earlier, 5.20 shows that 5.21 holds "uniformly."

Suppose $X$ is a set. We define $\hat{\Phi}_{X}^{\mu}(f)$ for each $\mu \in \mathcal{O}$ and each $f \in W^{\mu}(X)$ by induction as follows (5.22-5.24) so that $\hat{\Phi}_{X}^{\mu}(f)$ is a nonvoid subset of $\Im$.

5.22. If $f \in w^{0}(X)$, let $\hat{\Phi}_{X}^{\mu}(f)=\{0\}$.

5.23. Sup.pose $\mu>0$ is a nonlimit ordinal number. Consider $f \in w^{\mu}(X)$. If $f=0$, let $\hat{\Phi}_{X}^{\mu}(f)=\{0\}$. If $f \neq 0$, let

$$
\hat{\Phi}_{X}^{\mu}(f)=\left\{t \cup\{[\operatorname{dom}(t), \alpha]\} \mid \alpha \in \operatorname{dom}(f) ; t \in \hat{\Phi}_{X}^{\mu-1}(f(\alpha))\right\} .
$$

5.24. Suppose $\mu>0$ is a limit ordinal number. Consider $f \in w^{\mu}(X)$. Then let $\hat{\Phi}_{X}^{\mu}(f)=\hat{\Phi}_{X}^{\gamma}(f)$ where $\gamma$ is the first $\lambda<\mu$ such that $f \in w^{\lambda}(X)$ (cf. 2.4).

The discussion between 5.11 and 5.13 (including 5.12) could be repeated with " $\Phi$ " replaced by " $\hat{\Phi}$ ". The result is a function for each $\mu \in \mathcal{O}$.

5.25. Theorem. Suppose $\mu, \nu \in \mathcal{O}$. Suppose $f \in W^{\mu}(\mathcal{u})$, and suppose $f \in w^{\mu}\left(\mathcal{U}^{*}\right)$ if $\mu$ is infinite. Suppose $g \in w^{\nu}(\mathcal{u})$, and suppose $g \in W^{\nu}\left(\mathcal{u}^{*}\right)$ if $\nu$ is infinite. Suppose

Then $f=g$.

$$
\operatorname{im}\left(\Lambda^{\mu}\left(\Gamma^{\mu}(f)\right)\right)=\operatorname{im}\left(\Lambda^{\nu}\left(\Gamma^{\nu}(g)\right)\right), \quad \hat{\Phi}^{\mu}(f)=\hat{\Phi}^{\nu}(g) .
$$

Proof. By 5.20.3 we are supposing

$$
\operatorname{dom}\left(\Phi^{\mu}(f)\right)=\operatorname{dom}\left(\Phi^{\nu}(g)\right), \quad \hat{\Phi}^{\mu}(f)=\hat{\Phi}^{\nu}(g) .
$$

These equations will be easier to work with.

We proceed by induction on $\max (\mu, \nu)$. We may suppose $\mu \leqq \nu$.

Consider $\nu=0$. Then also $\mu=0$, and, by 5.9,

$$
\{[0, f]\}=\operatorname{dom}\left(\Phi^{0}(f)\right)=\operatorname{dom}\left(\Phi^{0}(g)\right)=\{[0, g]\} .
$$

Hence $f=g$.

Consider $\nu>0$ a nonlimit ordinal and $\mu=0$. By 5.9,

$$
\{[0, f]\}=\operatorname{dom}\left(\Phi^{0}(f)\right)=\operatorname{dom}\left(\Phi^{\nu}(g)\right) \text {. }
$$

By 5.10, for some $t \in J, x \in X$, and $\alpha \in \mathcal{O}$,

$$
[0, f]=[t \cup\{[\operatorname{dom}(t), \alpha]\}, x], \quad 0=t \cup\{[\operatorname{dom}(t), \alpha]\} \neq 0,
$$

a contradiction. Thus $\mu>0$ if $\nu>0$.

Consider $\nu$ and $\mu>0$ nonlimit ordinals. By 5.23 (since $\Phi^{\lambda}(h) \subset J$ is nonvoid for each $\lambda \in \mathcal{O}$ and each $h \in \operatorname{dom}\left(\hat{\Phi}^{\lambda}\right)$ ), 


$$
\begin{aligned}
\operatorname{dom}(f) & =\left\{\alpha \mid t \cup\{[\operatorname{dom}(t), \alpha]\} \in \hat{\Phi}^{\mu}(f) \text { for some } t \in J\right\} \\
& =\left\{\alpha \mid t \cup\{[\operatorname{dom}(t), \alpha]\} \in \hat{\Phi}^{\nu}(g) \text { for some } t \in J\right\}=\operatorname{dom}(g) .
\end{aligned}
$$

For each $\alpha \in \operatorname{dom}(f)$, let

$$
S(\alpha, f)=\left\{[t \cup\{[\operatorname{dom}(t), \alpha]\}, x] \mid[t, x] \in \operatorname{dom}\left(\Phi^{\mu-1}(f(\alpha))\right)\right\},
$$

and let $S(\alpha, g)$ be defined similarly. By 5.10,

$$
\bigcup_{\alpha \in \operatorname{dom}(f)} S(\alpha, f)=\operatorname{dom}\left(\Phi^{\mu}(f)\right)=\operatorname{dom}\left(\Phi^{\nu}(g)\right)=\bigcup_{\alpha \in \operatorname{dom}(\theta)} S(\alpha, g) .
$$

It follows easily that $S(\alpha, f)=S(\alpha, g)$, and then also that

$$
\operatorname{dom}\left(\Phi^{\mu-1}(f(\alpha))\right)=\operatorname{dom}\left(\Phi^{\nu-1}(g(\alpha))\right) \text {, }
$$

for each $\alpha \in \operatorname{dom}(f)=\operatorname{dom}(g)$. Moreover, for each $\alpha \in \operatorname{dom}(f)$,

$$
\begin{aligned}
\hat{\Phi}^{\mu-1}(f(\alpha)) & =\left\{t \in \mathfrak{J} \mid t \cup\{[\operatorname{dom}(t), \alpha]\} \in \hat{\Phi}^{\mu}(f)\right\} \\
& =\left\{t \in \mathfrak{J} \mid t \cup\{[\operatorname{dom}(t), \alpha]\} \in \hat{\Phi}^{\gamma}(g)\right\}=\hat{\Phi}^{\mu-1}(g(\alpha)) .
\end{aligned}
$$

Hence, by the induction hypothesis, $f(\alpha)=g(\alpha)$ for each $\alpha \in \operatorname{dom}(f)$. Hence $f=g$.

Consider $\nu$ a nonlimit ordinal and $\mu$ a limit ordinal. Let $\gamma$ be the first ordinal $\lambda<\mu$ for which $f \in w^{\lambda}\left(\mathcal{U}^{*}\right)$. Then (cf. 5.11 and 2.8$) \Phi^{\nu}(g)=\Phi^{\mu}(f)$ $=\Phi^{\gamma}(f)$. By 5.24 and the previously mentioned analogue of $5.12, \hat{\Phi}^{\nu}(g)=\hat{\Phi}^{\mu}(f)$ $=\hat{\Phi}^{\gamma}(f)$. Moreover, by $2.4, \gamma$ is not a limit ordinal. Hence $f=g$ by the preceding paragraph.

The induction step for $\nu$ a nonlimit ordinal is complete. The induction step for $\nu$ a limit ordinal is similar to the argument in the preceding paragraph; the details will be omitted.

The induction is complete. Q.e.d.

Consider any set $X$. Let

$$
\hat{W}(X)=\{f \in W(X) \mid \text { if } \operatorname{dom}(f) \text { has a largest member, say } \beta \text {, then } f(\beta) \neq 0\} \text {. }
$$

Define $\hat{W}^{\mu}(X)$ for $\mu \in \mathcal{O}$ in the obvious way (cf. 2.1-2.5); $\hat{W}^{\mu}(X) \subset \mathfrak{W}^{\mu}(X)$ for each $\mu \in \mathcal{O}$. Analogues of 2.5-2.7 with "W" replaced by " $\hat{W}$ " may be proved, and $\hat{\mathcal{W}}(\alpha)$ may be defined for each class $\alpha$ in the obvious way. In 5.25 , if "W" is replaced throughout by "Wै," the hypothesis that $\hat{\Phi}^{\mu}(f)=\hat{\Phi}^{\prime}(g)$ may be dropped (examine the proof of 5.25).

One may effectively define by known methods (the details will be omitted; cf. [6]) a one-one function $\Delta$ from $J$ onto $\theta$ such that

$$
\operatorname{im}\left(\Delta \mid J_{\omega^{\alpha}}\right)=\omega^{\alpha}
$$

There is a one-one function $\Delta^{*}$ from $P(\mathcal{J})$ onto $\mathcal{P}(\mathcal{O})$ such that $\Delta^{*}(A)$ $=\{\Delta(t) \mid t \in A\}$ for each $A \in \mathcal{P}(\mathfrak{\jmath})$. Observe that

$$
\operatorname{im}\left(\Delta^{*} \mid \rho\left(J_{\omega^{\alpha}}\right)\right)=\rho\left(\omega^{\alpha}\right) \quad(\alpha \in \mathcal{O}) .
$$


There is a one-one function $\Delta^{*}$ from $\mathfrak{J} \times \mathcal{u}$ onto $\mathcal{O} \times \mathfrak{u}$ such that $\Delta^{*}([t, u])$ $=[\Delta(t), u]$ for all $t \in J$ and $u \in \mathcal{u}$. For each $\mu \in \mathcal{O}$ there is a function $\boldsymbol{\Xi}^{\mu}$ with domain $w^{\mu}\left(\mathcal{U}_{1}\right), \mathfrak{u}_{1}$ being $\mathcal{u}$ or $\mathfrak{u}^{*}$ according as $\mu$ is finite or infinite, such that

$$
\Xi^{\mu}(f)=\left[\Delta^{*}\left(\hat{\Phi}^{\mu}(f)\right), \operatorname{im}\left(\Delta^{*} \circ\left(\Lambda^{\mu}\left(\Gamma^{\mu}(f)\right)\right)\right)\right] \quad\left(f \in \mathcal{u}_{1}\right) .
$$

The following theorem follows from 5.25; for more information cf. 6.11.

5.26. Theorem. For each ordinal number $\mu$, $\Xi^{\mu}$ is a one-one function from $w^{\mu}\left(\mathcal{U}_{1}\right)$ to $\mathcal{P}(\mathcal{O}) \times \mathcal{P}\left(\mathcal{O} \times \mathcal{U}_{1}\right)$ where $\mathfrak{u}_{1}$ is $\mathcal{u}$ or $\mathfrak{u}^{*}$ according as $\mu$ is finite or infinite.

5.27. We outline briefly other developments. One may define by induction functions spt ${ }^{\mu}$ and $\sigma^{\mu}$ from $W^{\mu}\left(\mathcal{U}^{*}\right)$ to $\mathcal{O}$ such that 5.27.1-5.27.4 below hold. More precisely, first define spt $t_{X}^{\mu}$ and $\sigma_{X}^{\mu}$ on $w^{\mu}(X)$ for each set $X \subset \mathcal{U}^{*}$, and let

$$
s p t^{\mu}=\bigcup_{x \in \mathcal{P}\left(\mathcal{U}^{*}\right)} s p t_{X}^{\mu}, \quad \sigma^{\mu}=\bigcup_{x \in \mathcal{P}\left(\mathcal{U}^{*}\right)} \sigma_{X}^{\mu} .
$$

5.27.1. For each $f \in \mathcal{u}^{*}=w^{0}\left(\mathcal{u}^{*}\right), \operatorname{spt}^{0}(f)=\sigma^{0}(f)=1$.

5.27.2. For each $f \in W^{1}\left(\mathcal{U}^{*}\right), s p t^{1}(f)=\sigma^{1}(f)=\operatorname{dom}(f)$.

5.27.3. For each $f \in w^{\mu+1}\left(\mathcal{u}^{*}\right)$,

$$
\begin{aligned}
s p t^{\mu+1}(f) & =[\operatorname{dom}(f)] \cup\left[\bigcup_{\alpha \in \operatorname{dom}(f)} s p t^{\mu}(f(\alpha))\right], \\
\sigma^{\mu+1}(f) & =\sum_{\alpha \in \operatorname{dom}(f)} \sigma^{\mu}(f(\alpha)) .
\end{aligned}
$$

5.27.4. If $\mu$ is a limit ordinal number and $f \in w^{\mu}\left(\mathcal{U}^{*}\right)$, then, where $\gamma$ is the first ordinal number $\lambda$ for which $f \in w^{\lambda}\left(\mathcal{U}^{*}\right), \operatorname{spt}^{\mu}(f)=\operatorname{spt}^{\gamma}(f)$ and $\sigma^{\mu}(f)=\sigma^{\gamma}(f)$. It is routine to prove 5.27.5-5.27.10 below.

5.27.5. If $f \in w^{\mu}\left(\mathcal{U}^{*}\right) \cap w^{\nu}\left(\mathcal{u}^{*}\right)$, then $\operatorname{spt}^{\mu}(f)=\operatorname{spt^{\nu }}(f) \leqq \sigma^{\nu}(f)=\sigma^{\mu}(f)$.

5.27.6. If $\phi$ is a function from $X \subset \mathcal{U}^{*}$ to $Y \subset \mathcal{U}^{*}$, then for each $f \in w^{\mu}(X)$, $s p t^{\mu}\left(\phi^{\mu}(f)\right) \leqq s p t^{\mu}(f)$ and $\sigma^{\mu}(\phi(f)) \leqq \sigma^{\mu}(f)$.

5.27.7. If $f \in w^{\mu}\left(\mathcal{U}^{*}\right)$, then $\operatorname{dom}\left(\Lambda^{\mu}(f)\right) \leqq \sigma^{\mu}(f)$, and $\operatorname{dom}\left(\Lambda^{\mu}(f)\right)=\sigma^{\mu}(f)$ if $f$ is free.

5.27.8. If $f \in w^{\mu}\left(\mathcal{u}^{*}\right)$, then

$$
\operatorname{dom}\left(\Lambda^{\mu}\left(\Gamma^{\mu}(f)\right)\right)=\sigma^{\mu}\left(\Gamma^{\mu}(f)\right)=\sigma^{\mu}(f),
$$

and

$$
s p t^{\mu}\left(\Gamma^{\mu}(f)\right)=s p t^{\mu}(f) .
$$

5.27.9. If $f \in w^{\mu}(X)$ with $X \subset \mathcal{u}^{*}$, then $\Gamma^{\mu}(f) \in w^{\mu}(T, X X)$ where $\nu=s p t^{\mu}(f)$.

5.27.10. If $f \in w^{\mu}(X)$ with $X \subset \mathcal{U}^{*}$, then $\Xi^{\mu}(f) \in \mathcal{P}\left(\omega^{\beta}\right) \times \mathcal{P}\left(\omega^{\beta} \times X\right)$ where $\beta$ is the smallest ordinal number $\alpha$ for which $s p t^{\mu}(f) \leqq \omega^{\alpha}$.

6. Powers and cardinals arising from $w^{\mu}(X)$. We define by induction a function $\Psi$, whose domain is the class of all ordinal numbers, such that 


$$
\Psi(\mu)=\{[\lambda, \Psi(\lambda)] \mid \lambda<\mu\}
$$

for each ordinal number $\mu$. In particular, $\Psi(0)=0$. Then for each ordinal number $\mu, \Psi(\mu)$ is a function with domain $\mu$. If $\lambda<\mu$, then

$$
\operatorname{dom}(\Psi(\lambda))=\lambda \neq \mu=\operatorname{dom}(\Psi(\mu)),
$$

and hence $\Psi(\lambda) \neq \Psi(\mu)$. Thus $\Psi$ is one-one, and, for each ordinal number $\mu$, $\Psi(\mu)=\Psi \mid \mu$ is one-one. The following lemma may be proved by induction on $\mu$. We omit the details.

6.1. LEMMA. Suppose $X$ is a set, and suppose $\mu$ and $\nu$ are ordinal numbers with $\mu<\nu$. Then $\Psi(\mu) \in w^{\nu}(X)$.

The following lemma follows from 4.5, 4.10.4, and 4.10.3.

6.2. Lemma. Suppose $X$ and $Y$ are sets with $|X| \leqq|Y|$ (resp., $|X|=|Y|$ ). Then for each ordinal number $\mu,\left|w^{\mu}(X)\right| \leqq\left|w^{\mu}(Y)\right|$ (resp., $\left|w^{\mu}(X)\right|$ $\left.=\left|w^{\mu}(Y)\right|\right)$.

The following lemma would remain valid if $w(X)$ were replaced by $\{Y \subset X \mid$ there is a relation which well-orders $Y\}$; this was proved by Tarski $[7$, Theorem 10]. Moreover, this substitution for $w(X)$ would also be valid in 6.4 .

6.3. LemMA. For each set $X,|X|<|W(X)|$.

Proof. Use $\Lambda_{X}^{0}$ (cf. 3.1) to see that $|X| \leqq|W(X)|$. Suppose $|X|=|W(X)|$. Then there is a one-one function $\phi$ from $w(X)$ to $X$. Let $G$ be the set of all $f \in W(X)$ such that for each $\alpha \in \operatorname{dom}(f), f(\alpha)=\phi(f \mid \alpha)$. Let $g=U_{f \in G} f$. Then $g \in G$, and $g$ is the maximum member of $G$. Moreover, $\phi(g) \notin \operatorname{im}(g)$. Let

$$
h=g \cup\{[\operatorname{dom}(g), \phi(g)]\} \text {. }
$$

Then $h \in G$ contrary to $g$ being the maximum member of $G$. Hence $|X|$ $\neq|w(X)|$. Q.e.d.

6.4. REMARK. By combining the method of proof of 6.3 with a method of Specker [6], much more than 6.3 could be proved; e.g., $|X|^{2}<|w(X)|$ if $X$ is infinite.

6.5. Theorem. For each set $X$ and all ordinal numbers $\mu$ and $\nu, 6.5 .1-$ 6.5.3 below hold.

6.5.1. If $\mu<\nu$, then $\left|w^{\mu}(X)\right|<\left|w^{\nu}(X)\right|$.

6.5.2. $\left|w^{\mu+\nu}(X)\right|=\left|w^{\nu}\left(w^{\mu}(X)\right)\right|$.

6.5.3. $|\mu| \leqq\left|w^{\mu}(X)\right|$.

One may prove 6.5.1 by induction on $\nu$ using 2.1-2.4 and 6.2. The details will be omitted.

Proof of 6.5.2. By 2.5, 


$$
\left|w^{\nu}\left(w^{\mu}(X)\right)\right| \leqq\left|w^{\mu+\nu}(X)\right| \text {, }
$$

equality holding if $\nu$ is finite or $\mu$ is 0 or $\mu$ is a limit ordinal. Suppose $\nu$ is infinite. There are ordinal numbers $\lambda$ and $\delta$ such that $\mu=\lambda+\delta, \mu$ is 0 or a limit ordinal, and $\delta$ is finite. Then $\delta+\nu=\nu$, and $\left|w^{\lambda}(X)\right| \leqq\left|w^{\mu}(X)\right|$ by 6.5.1. Hence, by the cases of equality already established and 6.2 ,

$$
\begin{aligned}
\left|w^{\mu+\nu}(X)\right| & =\left|w^{\lambda+\delta+\nu}(X)\right|=\left|w^{\lambda+r}(X)\right| \\
& =\left|W^{\nu}\left(w^{\lambda}(X)\right)\right| \leqq\left|W^{\nu}\left(w^{\mu}(X)\right)\right| .
\end{aligned}
$$

Now 6.5.2 holds by the Schröder-Bernstein equivalence theorem. Q.e.d.

Proof of 6.5.3. By 6.1, $\Psi(\mu) \in w^{\mu+1}(X)$. Hence $\operatorname{im}(\Psi(\mu)) \subset w^{\mu}(X)$, and, since $\Psi(\mu)=\Psi \mid \mu$ is one-one, 6.5 .3 holds. Q.e.d.

6.6. REMARK. One can obviously strengthen 6.5 .3 for nonlimit ordinals. In the proof of 6.5.3, observe that

$$
\{[\Psi(\mu)] \circ f \mid f \in W(\mu) \text { is increasing }\} \subset W^{\mu+1}(X)
$$

and hence $2^{|\mu|} \leqq W^{\mu+1}(X)$.

Consider any set $X$. After F. Hartogs [3] we define

$$
\aleph(X)=\sup \{\operatorname{dom}(f) \mid f \in W(X)\}=\bigcup_{f \in W_{(X)}} \operatorname{dom}(f) .
$$

For the elementary properties of $\aleph(X)$, cf. $[3 ; 4]$; we mention a few. $\aleph(X)$ is a cardinal number. If $X$ is not finite, $\aleph(X)$ is the smallest cardinal number (and smallest ordinal number) not equi-potent with a subset of $X$. If $X$ is finite, $|N(X)|=|X|$. We have the inequalities (sharpened later in 6.13)

$$
|\aleph(X)| \leqq^{*} 2^{|x|^{2}}, \quad|\aleph(X)| \leqq^{*} 2^{2^{|x|}}
$$

and hence

$$
2^{|k(x)|} \leqq 2^{2^{|x|^{2}}}, \quad 2^{|k(x)|} \leqq 2^{2^{2|x|}} .
$$

For each ordinal number $\mu, \boldsymbol{N}\left(\boldsymbol{N}_{\mu}\right)=\boldsymbol{\aleph}_{\mu+1}$. Consider any set $Y$ in addition to $X$. If $X$ or $Y$ is infinite, then

$$
\aleph(X \times Y)=\aleph(X \cup Y)=\max (\boldsymbol{N}(X), \boldsymbol{N}(Y)) .
$$

If $|X| \leqq|Y|$ (resp., $|X|=|Y|$ ), then $\aleph(X) \leqq \aleph(Y)$ (resp., $\aleph(X)=\aleph(Y)$ ).

\subsection{Theorem. For each infinite set $X, \aleph(W(X)) \leqq \aleph(\odot(X))$.}

Proof. Consider any infinite $f \in w^{2}(X)$. Let $A=\operatorname{im}\left(\Lambda^{2}(f)\right)$. Then $A \subset X$ is equi-potent with the ordinal number $\operatorname{dom}\left(\Lambda^{2}(f)\right)$, and $A$ is infinite since $f \in W^{2}(A)$ is infinite. Hence $|w(A)|=|\odot(A)|$ by standard arguments, and, since $f \in W(W(A))$,

$$
\operatorname{dom}(f)<\aleph(W(A))=\aleph(P(A)) \leqq \aleph(P(X)) .
$$


Given any finite $f \in \mathcal{W}^{2}(X)$, trivially, $\operatorname{dom}(f)<\boldsymbol{N}(\odot(X))$. It follows that

$$
\aleph(W(X))=\bigcup_{f \in W^{2}(X)} \operatorname{dom}(f) \leqq \aleph(\mathcal{P}(X)) .
$$

Q.e.d.

It is elementary that $|\mathcal{N}(X)| \$|X|$ if $X$ is an infinite set. The following lemma is a routine consequence.

6.8. Lemma. Suppose $X$ and $Y$ are infinite sets with $|X| \leqq|Y|$. Then $\aleph(X)=\aleph(Y)$ if and only if $|\boldsymbol{\aleph}(X)| \$|Y|$.

For each set $X \subset \mathcal{u}^{*}$ and each ordinal number $\mu>0$ we let

$$
\mathcal{N}^{\mu}(X)=\underset{f \in \mathcal{W}^{\mu}(X) \backslash X}{U} \operatorname{dom}(f),
$$

and we let $\aleph^{\circ}(X)=1$. For each set $X \nsubseteq \mathcal{U}^{*}$ and each ordinal number $\mu$, we observe that $\{0\} \times X$ is equi-potent with $X$ and is $\subset u^{*}$ by 5.7 , and we let $\boldsymbol{N}^{\mu}(X)=\boldsymbol{N}^{\mu}(\{0\} \times X)$ [this equation holds also if $X \subset \boldsymbol{u}^{*}$ by the next assertion]. Observe from 4.10.4 that if $X$ and $Y$ are equi-potent sets, then $\aleph^{\mu}(X)$ $=\aleph^{\mu}(Y)$ for each ordinal number $\mu$. It is easy to prove that for each set $X$, $\aleph^{1}(X)=\boldsymbol{N}(X)$, and $\boldsymbol{N}^{\mu}(X)$ is a cardinal number for each ordinal number $\mu$.

6.9. REMARK. Suppose $X$ is a set. For each ordinal number $\mu$,

$$
\aleph^{\mu+1}(X)=\aleph\left(W^{\mu}(X)\right)=\aleph^{1}\left(\text { WO }^{\mu}(X)\right) .
$$

For each limit ordinal number $\mu$,

$$
\aleph^{\mu}(X)=\bigcup_{\lambda<\mu} \aleph^{\lambda}(X)=\sup _{\lambda<\mu} \aleph^{\lambda}(X) .
$$

If $\mu<\nu \in \mathcal{O}$, then $\aleph^{\mu}(X) \leqq \boldsymbol{N}^{\prime}(X)$.

6.10. Theorem. Suppose $X$ and $Y$ are sets such that $\boldsymbol{\aleph}(X) \leqq \aleph(Y)$ (resp., $\boldsymbol{N}(X)=\boldsymbol{\aleph}(Y))$. Then for each ordinal number $\mu, \boldsymbol{\aleph}^{\mu}(X) \leqq \boldsymbol{\aleph}^{\mu}(Y)\left(\right.$ resp., $\boldsymbol{\aleph}^{\mu}(X)$ $\left.=\boldsymbol{N}^{\mu}(Y)\right)$.

Proof. (The result for $\aleph(X)=\aleph(Y)$ follows immediately from the result for $\boldsymbol{\aleph}(X) \leqq \aleph(Y)$.) We may suppose $X \cup Y \subset \mathcal{U}^{*}$. The case $\mu=0$ is trivial. Consider an ordinal number $\mu>0$. Consider $f \in W^{\mu}(X) \backslash X$. Then $\Lambda^{\mu}(f)$ $\in W(X)$. Since $\boldsymbol{N}(X) \leqq \aleph(Y), \operatorname{dom}\left(\Lambda^{\mu}(f)\right)=\operatorname{dom}(g)$ for some $g \in w(Y)$. Let $\phi=g \circ\left(\left(\Lambda^{\mu}(f)\right)^{-1}\right)$. Then $\phi$ is a one-one function with domain $A=\operatorname{im}\left(\Lambda^{\mu}(f)\right)$ $\subset X$ and image $\operatorname{im}(g) \subset Y$, and $f \in w^{\mu}(A)$ by 3.8. Then $\phi^{\mu}(f) \in w^{\mu}(Y) \backslash Y$ (cf. 2.1 and 4.8), and $\operatorname{dom}\left(\phi^{\mu}(f)\right)=\operatorname{dom}(f)$ by 4.10.4. Hence

Q.e.d.

$$
\begin{aligned}
\aleph^{\mu}(X) & =\underbrace{U}_{f \in \mathcal{W}^{\mu}(X) \backslash X} \operatorname{dom}(f) \\
& \bigcup_{\rho \in \mathbb{W}^{\mu}(Y) \backslash Y} \operatorname{dom}(g)=\aleph^{\mu}(Y) .
\end{aligned}
$$


The following theorem follows from 5.26 and 5.27 .10 (cf. also the last part of 6.9).

6.11. Theorem. Suppose $X \subset \mathcal{U}^{*}$ and $\mu \in \mathcal{O}$, and suppose $X$ or $\mu$ is infinite. Then $\mathbb{G}^{\mu}$ maps $w^{\mu}(X)$ one-one into $P\left(\boldsymbol{N}^{\mu}(X)\right) \times P\left(\boldsymbol{N}^{\mu}(X) \times X\right)$.

6.12. Corollary. Suppose $0 \neq X \in \odot\left(\mathcal{u}^{*}\right)$ and $\mu \in \mathcal{O}$, and suppose $X$ or $\mu$ is infinite. Then

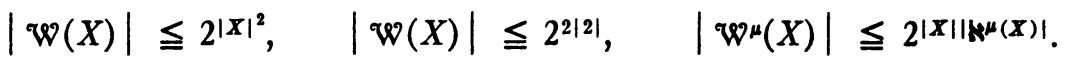

[The inequations for $|w(X)|$ hold for all sets $X$.]

Proof. There are one-one functions $\phi_{1}$ and $\phi_{2}$ from $w(X)$ into $\odot(X \times X)$ and $P(P(X))$ respectively such that

$$
\begin{array}{ll}
\phi_{1}(f)=\{[f(\alpha), f(\beta)] \mid \alpha<\beta \in \operatorname{dom}(f)\} & (f \in W(X)), \\
\phi_{2}(f)=\{\{f(\alpha) \mid \alpha<\beta\} \mid \beta \leqq \operatorname{dom}(f)\} & (f \in W(X)) .
\end{array}
$$

The inequations for $|w(X)|$ are thus established. If $X$ is infinite, $\aleph^{\mu}(X) \geqq \aleph_{0}$. If $\mu$ is infinite, $\aleph^{\mu}(X) \geqq \mu \geqq \aleph_{0}$ by 6.1 . Hence $\aleph^{\mu}(X) \geqq \aleph_{0}$. By 6.11,

$$
\left|W^{\mu}(X)\right| \leqq 2^{\left|\mathbb{K}^{\mu}(X)\right|} 2^{|X|\left|\mathbb{k}^{\mu}(X)\right|}=2^{(|X|+|1|)\left|\aleph^{\mu}(X)\right|}=2^{|X|\left|\mathbb{k}^{\mu}(X)\right|} .
$$

Q.e.d.

For each $\mu \in \mathcal{O}$ there is a function $\tilde{\Xi}^{\mu}$ with domain $u_{1}=\operatorname{dom}\left(\Xi^{\mu}\right)$ such that

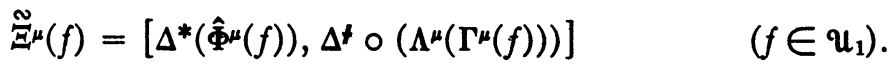

It is easy to prove analogues of 5.26 and 6.11 obtained by replacing " $\Xi^{\mu}$ " by

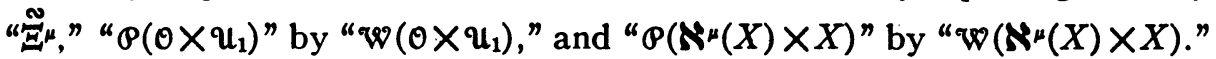

6.13. Lemma. Suppose $X$ is an infinite set. Then

$$
\begin{aligned}
& |X|^{2}|\mathcal{N}(X)| \leqq 2^{|x|^{2}}, \quad 2^{|X|^{2}|M(X)|} \leqq 2^{2^{|x|^{2}}}, \\
& 2^{|X|}|\mathbb{N}(X)| \leqq * 2^{2^{|X|}}, \quad 2^{2^{|x|}|\xi(X)|} \leqq 2^{2^{2^{|x|}}} .
\end{aligned}
$$

Proof. Let $\phi_{1}$ and $\phi_{2}$ be the functions in the proof of 6.12 . There is a function $\psi_{1}$ from $\rho(X \times X)$ onto $[X \times X] \times[\aleph(X) \backslash 2]$ such that for all $f \in W(X)$ with domain $>1$ and all $x, y \in X \backslash \operatorname{im}(f)$,

$$
\psi_{1}\left(\{[x, y]\} \cup \phi_{1}(f)\right)=[[x, y], \operatorname{dom}(f)] .
$$

(E.g., if $S$ is the set of all subsets of $X \times X$ not of the form $\{[x, y]\} \cup \phi_{1}(f)$ with $f, x, y$ as just specified, let $\psi_{1} \mid S$ be constant, the constant value of $\psi_{1} \mid S$ being any member of $[X \times X] \times[N(X) \backslash 2]$.) Since $|\aleph(X) \backslash 2|=|\aleph(X)|$, the first inequality is established. The second inequality is a consequence of the first. There is a function $\psi_{2}$ from $\rho(\rho(X))$ onto $\rho(X) \times[\aleph(X) \backslash 2]$ such that for all $f \in W(X)$ with domain $>1$ and all $Y \subset X$,

$$
\psi_{2}\left(\{\{y\} \mid y \in Y\} \cup\left(\phi_{2}(f) \backslash\{f(0)\}\right)\right)=[Y, \operatorname{dom}(f)] .
$$


(Cf. the parenthetical remark made in connection with $\psi_{1 .}$.) Since $|\aleph(X) \backslash 2|$ $=|\boldsymbol{\aleph}(X)|$, the third inequality is established. The fourth inequality follows from the third. Q.e.d.

Suppose $\alpha$ and $\mu$ are ordinal numbers. Then $\aleph_{\alpha}$ is $\mu$-accessible if and only if

$$
\aleph_{\alpha}=\bigcup_{\lambda \in \mu} \phi(\lambda)
$$

for some nondecreasing function $\phi$ from $\mu$ into $\boldsymbol{N}_{\alpha}$.

6.14. TheOREM. Suppose $X$ is an infinite set such that $\aleph^{2}(X)=\aleph^{1}(X)$. Then there is a first ordinal number $\mu>2$ for which $\aleph^{\mu}(X) \neq \aleph^{1}(X)$, say $\mu=\nu$. Then $\nu<\aleph(X), \nu$ is not a limit ordinal, $\nu-1$ is a limit ordinal, and $\aleph(X)$ is $(\nu-1)$-accessible. Moreover, for each $\mu<\nu$,

$$
\begin{aligned}
& \left|w^{\mu}(X)\right| \leqq 2^{|X| \cdot\left|X_{\mu}(X)\right|} \leqq 2^{2^{|X|^{2}}}, \\
& \left|w^{\mu}(X)\right| \leqq 2^{2^{2^{2}|X|}}
\end{aligned}
$$

Proof. We may suppose $X \subset \mathcal{u}^{*}$. The existence of $\nu$ and $\nu<\aleph(X)$ follow from 6.5.3. Moreover, $\aleph(X)=\operatorname{dom}(g)$ for some $g \in w^{\nu}(X)$, say for $g=g_{0}$.

Suppose $\nu$ is a limit ordinal. Then for some nonlimit ordinal $\mu>0$, $g_{0} \in W^{\mu}(X)$, and

$$
\boldsymbol{\aleph}(X)=\aleph^{\mu}(X)>\operatorname{dom}\left(g_{0}\right)=\boldsymbol{N}(X),
$$

a contradiction.

Thus $\nu$ is not a limit ordinal.

If $\nu-1$ is not a limit ordinal, then

$$
\boldsymbol{N}\left(W^{-2}(X)\right)=\aleph^{-1}(X)=\aleph(X)
$$

and hence, by 6.10 ,

$$
\begin{aligned}
\aleph^{\nu}(X) & =\aleph\left(W^{p-1}(X)\right)=\aleph\left(W^{1}\left(W^{p-2}(X)\right)\right) \\
& =\aleph^{2}\left(W^{v-2}(X)\right)=\aleph^{2}(X)=\aleph(X),
\end{aligned}
$$

a contradiction.

Thus $\nu-1$ is a limit ordinal.

Since $\boldsymbol{N}(X)<\boldsymbol{N}(X)=\boldsymbol{N}\left(\mathbb{W}^{\nu-1}(X)\right), \boldsymbol{N}(X)=\operatorname{dom}(f)$ for some

$$
\left.f \in W_{\left(W^{p-1}\right.}(X)\right)=W\left(\bigcup_{\mu<\nu-1} w^{\mu}(X)\right) \text {. }
$$

Consider such an $f$. For each $\mu<\nu-1$ there is a unique strictly increasing function $\xi_{\mu}$ with domain an ordinal number $\delta_{\mu}$ and with

$$
\operatorname{im}\left(\xi_{\mu}\right)=\left\{\lambda \in \operatorname{dom}(f) \mid f(\lambda) \in \mathfrak{w}^{\mu}(X) ; f(\lambda) \notin w^{\gamma}(X) \text { for each } \gamma<\mu\right\} .
$$

Then $f \circ \xi_{\mu} \in W^{\mu+1}(X)$ for each $\mu<\nu-1$. There is a unique function $g \in W^{\nu}(X)$ with domain 


$$
\delta=\sum_{\mu<\nu-1} \delta_{\mu}
$$

such that for each $\mu<\nu-1$ and each $\lambda<\delta_{\mu}$,

$$
g\left(\left[\sum_{\gamma<\mu} \delta_{\gamma}\right]+\lambda\right)=f\left(\xi_{\mu}(\lambda)\right) .
$$

Then $\operatorname{im}(g)=\operatorname{im}(f)$, and hence $|\delta|=|\boldsymbol{N}(X)|$.

We shall prove that for each $\mu<\nu-1, \epsilon_{\mu}<\boldsymbol{N}(X)$ where $\epsilon_{\mu}=\sum_{\gamma<\mu} \delta_{\gamma}$. Suppose not. Then the inequality fails for some smallest $\mu<\nu-1$, say $\mu=\tau$. If $\tau$ is a limit ordinal, then $g \mid \epsilon_{\tau} \in W^{++1}(X)$, and hence $\epsilon_{\tau}\left\langle\aleph^{++1}(X)=\aleph(X)\right.$, a contradiction. If $\tau$ is not a limit ordinal, then $\epsilon_{\tau-1}<\aleph(X)$ and $\delta_{\tau-1}<\aleph(X)$, hence $\epsilon_{\tau}=\epsilon_{\tau-1}+\delta_{\tau-1}<\boldsymbol{N}(X)$, a contradiction. The inequality $\epsilon_{\mu}<\boldsymbol{N}(X)$ for all $\mu<\nu-1$ is now established.

Since $|\boldsymbol{N}(X)|=|\delta|$ where $\delta=U_{\mu<\nu-1} \epsilon_{\mu}$ with $\epsilon_{\mu}<\aleph(X)$ for each $\mu<\nu-1$, $\aleph(X)=\delta$. Hence also $\aleph(X)$ is $(\nu-1)$-accessible.

By 6.12 and 6.13 and the fact that $\aleph(X) \geqq \aleph_{0}$, for each $\mu<\nu$ we have

$$
\begin{aligned}
& \left|W^{\mu}(X)\right| \leqq 2^{|X||k(X)|} \leqq 2^{2^{|X|^{2}}}, \\
& \left|W^{\mu}(X)\right| \leqq 2^{|X||k(X)|} \leqq 2^{2^{2^{|X|}}} .
\end{aligned}
$$

Q.e.d.

7. On sufficient conditions for the axiom of choice. For each power $\mathfrak{m}$ and each ordinal number $\alpha$ let $H(\mathfrak{m} ; \alpha)$ be the statement that there is no function $\phi$ with domain $\alpha$ such that $\phi(\mu)$ is a set with $\mathfrak{m}<|\phi(\mu)|<2^{\mathfrak{m}}$ for each $\mu<\alpha$ and such that $|\phi(\mu)|<|\phi(\nu)|$ for all $\nu<\alpha$ and $\mu<\nu$. Loosely speaking, $H(\mathrm{~m} ; \alpha)$ is the statement that there is no strictly increasing $\alpha$-sequence of powers strictly between $\mathfrak{m}$ and $2^{\mathfrak{m}}$. In particular, $H(\mathfrak{m} ; 1)$ is the statement $H_{\mathfrak{m}}$ or $H(\mathfrak{m})$ of $[4 ; 5 ; 6]$. If $\alpha<\beta \in \mathcal{O}$, then $H(\mathfrak{m} ; \alpha)$ implies $H(\mathfrak{m} ; \beta)$.

For each ordinal number $\alpha$, we define acc $\left(\boldsymbol{\aleph}_{\alpha}\right)$, the accessibility of $\boldsymbol{\aleph}_{\alpha}$, to be the smallest ordinal number $\mu$ such that $\boldsymbol{\aleph}_{\alpha}$ is $\mu$-accessible. Then $\operatorname{acc}\left(\boldsymbol{\aleph}_{\alpha}\right)$ is an infinite cardinal number.

The following theorem further refines Specker's sharpening [6] of the Lindenbaum-Tarski-Sierpiński theorem $[4 ; 5]$.

7.1. Theorem. Suppose $X$ is an infinite set such that $H(|X|)$ and $H\left(2^{|X|} ; \operatorname{acc}(\mathcal{N}(X))\right)$ hold. Then $2^{|X|}=|\boldsymbol{N}(X)|$.

Proof. By a result of Specker [6], $|X|^{2}=X$ by $H(|X|)$. Hence $|X|$ $<|w(X)| \leqq 2^{|X|}$ by 6.3 and 6.12 . Hence $|w(X)|=2^{|x|}$ by $H(|X|)$.

Suppose $|\boldsymbol{N}(X)| 2^{|x|}$. Then $\boldsymbol{\aleph}^{2}(X)=\boldsymbol{N}(\mathscr{W}(X))=\boldsymbol{N}(P(X))=\boldsymbol{N}^{1}(X)$ by 6.9 and 6.8. Let $\nu$ be given by 6.14. Then $\nu-1 \geqq \operatorname{acc}(\mathcal{N}(X))$, and

$$
2^{|X|}=\left|w_{(X)}\right|<\left|w^{\lambda}(X)\right|<\left|w^{\mu}(X)\right| \leqq 2^{2|X|^{2}}=2^{2|X|}
$$

if $1<\lambda<\mu \leqq \nu-1$ by 6.14 and 6.5 .1 , contrary to $H\left(2^{|x|} ; \operatorname{acc}(\mathcal{N}(X))\right)$. 
Hence $|\boldsymbol{\aleph}(X)| \leqq 2^{|\boldsymbol{x}|}$. Hence $|\boldsymbol{\aleph}(X)|=2^{|\boldsymbol{x}|}$ by an argument used by Specker [6]. Q.e.d.

7.2. Corollary. Suppose $X$ is an infinite set such that $H(|X|)$ and $H\left(2^{|x|} ; \omega\right)$ hold. Then $2^{|x|}=\boldsymbol{\aleph}(X)$.

For each set $X$ we define by induction $\rho^{\mu}(X)$ for each ordinal number $\mu$ so that $\mathcal{P}^{0}(X)=X, \mathcal{P}^{1}(X)=P(X), \mathbb{P}^{\mu+1}(X)=\mathcal{P}^{(\mu}\left(\mathcal{P}^{\mu}(X)\right)$, and

$$
\rho^{\nu}(X)=\bigcup_{\mu<\nu} \rho^{\mu}(X)
$$

if $\nu$ is a limit ordinal. For each set $X,\left\{\mathcal{P}^{\mu}(X)\right\}_{\mu \in \mathcal{O}}$ and $\left\{\mathscr{W}^{\mu}(X)\right\}_{\mu \in \mathcal{O}}$ are families of sets whose powers are strictly increasing. The axiom of choice implies that $\left|\nabla^{\mu}(X)\right|=\left|w^{\mu}(X)\right|$ for each $\mu \in \mathcal{O}$ if $X$ is infinite. The writer has been unable to prove that this condition implies the axiom of choice. We present a partial result (7.3 and 7.4) after stating two axioms.

Let $u_{0}$ be the class of all atoms. Axiom (*) below is a form of the restrictive axiom and restricts any set to be built ultimately from atoms. Axiom $\left({ }^{*}\right)$ is equivalent to the usual statement of the restrictive axiom (if stated to allow for atoms): Each nonvoid class $Q$ has as a member either an atom or a set disjoint with $Q$.

AxIOM $\left({ }^{*}\right) . u=U_{[X, \mu] \in \mathcal{P}(น 0) \times \mathcal{O}} \mathbb{P}^{\mu}(X)$.

Axıм $\left(^{* *}\right)$. For each set $X \subset \mathcal{U}_{0}$ there is a function $\phi$ with domain $\odot(X) \backslash\{0\}$ such that $\phi(Y) \in Y$ for each nonvoid set $Y \subset X$.

Axiom (**) is equivalent to each set of atoms having a well-ordering. The axiom of choice reads the same as the axiom just stated with " $C u_{0}$ " deleted. Axiom (**) holds automatically if $u_{0}=0$, as is the case for the von NeumannBernays-Gödel type of set theory.

7.3. Theorem. Suppose Axioms $\left({ }^{*}\right)$ and $\left({ }^{* *}\right)$ hold, and suppose (1) and (2) below hold.

(1) $\left|w^{\mu}(X)\right|=\left|\rho^{\mu}(X)\right|$ for each set $X \subset \mathcal{U}^{*}$ and each $\mu \in \mathcal{O}$.

(2) For all sets $X$ and $Y$ and each $\mu \in \mathcal{O}$, if $\left|\rho^{\mu}(X)\right|<\left|\rho^{\mu}(Y)\right|$, then $|X|<|Y|$.

Then the axiom of choice holds.

Proof. Consider $X \in \mathcal{P}\left(\mathcal{u}^{*}\right)$. There are a set $A \subset \mathcal{U}_{0}$ and $\nu \in \mathcal{O}$ such that $X \subset \operatorname{Pr}(A)$, and $|A| \leqq\left|\aleph_{\alpha}\right|$ for some ordinal number $\alpha$. It may be proved by induction that $\left|\rho^{\lambda}(A)\right||Y| \leqq\left|\rho^{\lambda}(A \cup Y)\right|$ for each $\lambda \in \mathcal{O}$ and each set $Y \subset \mathcal{U} \backslash A$. Hence, for each $\mu \in \mathcal{O}$,

$$
\begin{aligned}
|X|\left|\aleph^{\mu}(X)\right| & \leqq\left|\operatorname{Pr}^{\nu}(A)\right|\left|\aleph^{\mu}(X)\right| \\
& \leqq\left|\operatorname{Pr}^{\nu}\left(A \cup \boldsymbol{\aleph}^{\mu}(X)\right)\right| \leqq\left|\operatorname{Pr}^{\nu}\left(\boldsymbol{\aleph}_{\beta}\right)\right|
\end{aligned}
$$

with $\beta$ depending on $\mu$, and by 6.12 ,

$$
\left|w^{\mu}(X)\right| \leqq 2^{|x|\left|\mathcal{K}^{\mu}(X)\right|} \leqq\left|\rho^{\nu+1}\left(\boldsymbol{N}_{\beta}\right)\right| .
$$


[So far neither (1) nor (2) has been used.] Now let $\mu=\nu+2$ to get

$$
\left|\beta^{\nu+1}(X)\right|<\left|\beta^{\mu}(X)\right|=\left|w^{\mu}(X)\right| \leqq\left|\beta^{\nu+1}\left(\boldsymbol{K}_{\beta}\right)\right|
$$

by (1) and then $|X| \leqq\left|\aleph_{B}\right|$ by (2). Since $X \in \mathcal{P}\left(\mathcal{U}^{*}\right)$ was arbitrary, the axiom of choice holds. Q.e.d.

7.4. REMARK. The previous theorem remains valid if (1) and (2) together are replaced by the single condition (3) below.

(3) For all sets $X, Y \subset \mathcal{U}^{*}$ and each $\mu \in \mathcal{O}$, if $\left|w^{\mu}(X)\right|<\left|\rho^{\mu}(Y)\right|$, then $|X|<|Y|$.

In fact, the previous theorem remains valid if (1) and (2) together are replaced by the single condition (4) below.

(4) For each set $X \subset \mathcal{U}^{*}$ and each $\alpha \in O$, there is $\mu \in O$ such that for each set $Y$ with $\left|w^{\mu}(X)\right|<\left|\rho^{\alpha}(Y)\right|$, it is true that $|X|<|Y|$.

Loosely speaking, (4) states that for each set $X \subset \mathcal{u}^{*}$ and each $\alpha \in \mathcal{O}$, we have uniformly for $|Y| \ngtr|X|$ that eventually $\left|w^{\mu}(X)\right| \varangle\left|\rho^{\alpha}(Y)\right|$ as $\mu$ increases through 0 .

\section{REFERENCES}

1. P. Bernays, $A$ system for axiomatic set theory. I-VII, J. Symb. Logic vol. 2 (1937) pp. 65-77; vol. 6 (1941) pp. 1-17; vol. 7 (1942) pp. 65-89; vol. 7 (1942) pp. 133-145; vol. 8 (1943) pp. 89-106; vol. 13 (1948) pp. 65-79; vol. 19 (1954) pp. 81-96.

2. S. Eilenberg and S. MacLane, General theory of natural equivalences, Trans. Amer. Math. Soc. vol. 58 (1945) pp. 231-294.

3. F. Hartogs, Über das Problem der Wohlordnung, Math. Ann. vol. 76 (1915) pp. 438-443.

4. A. Lindenbaum and A. Tarski, Communications sur les recherches de la theorie des ensembles, Comptes rendus de la Societé des Sciences et des Lettres de Varsovie vol. 19 (1926) pp. 229-330.

5. W. Sierpinski, L'hypothese generalisee du continu et l'axiome du choix, Fund. Math. vol. 33 (1945) pp. 137-168.

6. E. Specker, Verallgemeinerte Kontinuumshypothese und Auswahlaxiom, Arch. Math. vol. 5 (1954) pp. 332-337.

7. A. Tarski, On well-ordered subsets of any set, Fund. Math. vol. 32 (1939) pp. 176-183.

UNIVERSITY OF KANSAS,

LAWRENCE, KANSAS 